\title{
Numerical simulation of scour and backfilling processes around a circular pile in waves
}

Baykal, Cüneyt; Sumer, B. Mutlu; Fuhrman, David R.; Jacobsen, Niels Gjøl; Fredsøe, Jørgen

Published in:

Coastal engineering

Link to article, DOI:

10.1016/j.coastaleng.2017.01.004

Publication date:

2017

Document Version

Peer reviewed version

Link back to DTU Orbit

Citation (APA):

Baykal, C., Sumer, B. M., Fuhrman, D. R., Jacobsen, N. G., \& Fredsøe, J. (2017). Numerical simulation of scour and backfilling processes around a circular pile in waves. Coastal engineering, 122, 87-107.

https://doi.org/10.1016/j.coastaleng.2017.01.004

\section{General rights}

Copyright and moral rights for the publications made accessible in the public portal are retained by the authors and/or other copyright owners and it is a condition of accessing publications that users recognise and abide by the legal requirements associated with these rights.

- Users may download and print one copy of any publication from the public portal for the purpose of private study or research.

- You may not further distribute the material or use it for any profit-making activity or commercial gain

- You may freely distribute the URL identifying the publication in the public portal 


\title{
Numerical simulation of scour and backfilling processes around a circular pile in waves
}

\author{
C. Baykal ${ }^{1}$, B. M. Sumer ${ }^{2}$, D. R. Fuhrman ${ }^{2}$, N. G. Jacobsen ${ }^{3}$ and J. Fredsøe ${ }^{2}$
}

1: Technical University of Denmark, Department of Mechanical Engineering, Section for Fluid Mechanics, Coastal and Maritime Engineering, Nils Koppels Alle 403, DK-2800 Kgs. Lyngby, Denmark. (Present address: METU, Department of Civil Engineering, Ocean Engineering Research Center, Dumlupinar Blvd. 06800, Cankaya, Ankara, Turkey, E-mail: cbaykal@metu.edu.tr)

2: Technical University of Denmark, Department of Mechanical Engineering, Section for Fluid Mechanics, Coastal and Maritime Engineering, Nils Koppels Alle 403, DK-2800 Kgs. Lyngby, Denmark

3: Deltares, Department of Coastal Structures and Waves, Boussinesqweg 1, 2629HV Delft, The Netherlands

Keywords: scour; backfilling; monopile; sediment transport; morphology; waves; oscillatory flow; turbulence modeling

\section{Highlights:}

- tool to predict wave-induced scour and backfilling processes around a monopile

- lee-wake flow as a governing mechanism in scour and backfilling in waves

- significance of final wave conditions in the determination of equilibrium scour depths

- change in the structure of vortex shedding in waves compared to steady current

- disappearance of two counter-rotating vortices further downstream of the lee-wake in waves 


\section{Abstract}

This study continues the investigation of flow and scour around a vertical pile, reported by Roulund et al. (2005). Flow and scour/backfilling around a vertical pile exposed to waves are investigated by using a three-dimensional numerical model based on incompressible Reynolds averaged Navier-Stokes equations. The model incorporates (1) $k$ - $\omega$ turbulence closure, (2) vortex shedding processes, (3) sediment transport (both bed and suspended load), as well as (4) bed morphology. The numerical simulations are carried out for a selected set of test conditions of the laboratory experiments of Sumer et al. (1997, 2013a), and the numerical results are compared with those of the latter experiments. The simulations are carried out for two kinds of beds: rigid bed, and sediment bed. The rigid-bed simulations indicate that the vortex shedding for waves around the pile occurs in a "one-cell" fashion with a uniform shedding frequency over the height of the cylinder, unlike the case for steady current where a two-cell structure prevails. The rigidbed simulations further show that the horseshoe vortex flow also undergoes substantial changes in waves. The amplification of the bed shear stress around the pile (including the areas under the horseshoe vortex and the lee wake region) is obtained for various values of the KeuleganCarpenter number, the principal parameter governing the flow around the pile in waves. The present model incorporated with the morphology component is applied to several scenarios of scour and backfilling around a pile exposed to waves. In the backfilling simulations, the initial scour hole is generated either by a steady current or by waves. The present simulations indicate that the scour and backfilling in waves are solely governed by the lee-wake flow, in agreement with observations. The numerical model has proven successful in predicting the backfilling of scour holes exposed to waves. The results of the numerical tests indicate that the equilibrium depth of scour holes is the same for both the scour and the backfilling for a given KeuleganCarpenter number, in full agreement with observations. 


\section{Introduction}

Monopile foundations are commonly used in marine environments by the offshore wind industry. WindEurope (formerly known as European Wind Energy Association, EWEA) reports annual market share of monopole type substructure for offshore wind turbines as $88 \%$ of installed in 2016, more than $81 \%$, the overall share of monopiles among all installed substructures in Europe (WindEurope, 2017). Considering the fact that up-to 35\% of the installation cost is attributable to the foundation works of such structures (Byrne and Houlsby, 2003), predicting the time variation of the scour-hole geometry around these structures in ever changing flow conditions of marine environments stands out as an important engineering challenge for the design of these structures. The forces acting on the structure, scour protection, natural frequency and fatigue conditions of the structure, stability of energy transfer cables, maintenance and repair costs and economic lifetime of the structure -- essential elements of this design exercise -- are all affected by the scour geometry around these structures.

When a pile is placed on the bed in a steady current or in waves, the flow will experience substantial changes (Figure 1): (1) a horseshoe vortex is formed in front of the pile; (2) a vortex flow pattern (usually in the form of vortex shedding) is formed at the lee-side of the pile; (3) the streamlines contract at the sides of the pile (e.g., Sumer and Fredsøe, 2002, for a detailed account of these effects); and (4) a pair of counter-rotating vortices is formed at the lee wake further downstream of the pile (Kirkil and Constantinescu, 2005, Kirkil et al., 2005, and Baykal et al., 2015). If the bed is erodible, the end effect of these changes is mainly to increase the sediment transport, resulting in local scour around the pile (see. e.g., Whitehouse, 1998, Sumer and Fredsøe, 2002). By contrast, when a scoured bed around a pile (generated by currents, or by large waves) is exposed to relatively smaller waves, the scour hole will be backfilled (Sumer et al., 2013a).

Several engineering models (Nielsen and Hansen, 2007; Raaijmakers and Rudolph, 2008a-b; and Harris et al. 2010) have been developed with the purpose of predicting the time history of scour and backfilling around monopile foundations for large times (weeks, months, or years). The existing information on scour has been successfully incorporated in these models. This is not so, however, for the backfilling because of the massive lack of knowledge although recent works 
(Sumer et al., 2013a, Baykal et al., 2014, Sumer et al., 2014) has shed some light onto the understanding of backfilling processes.

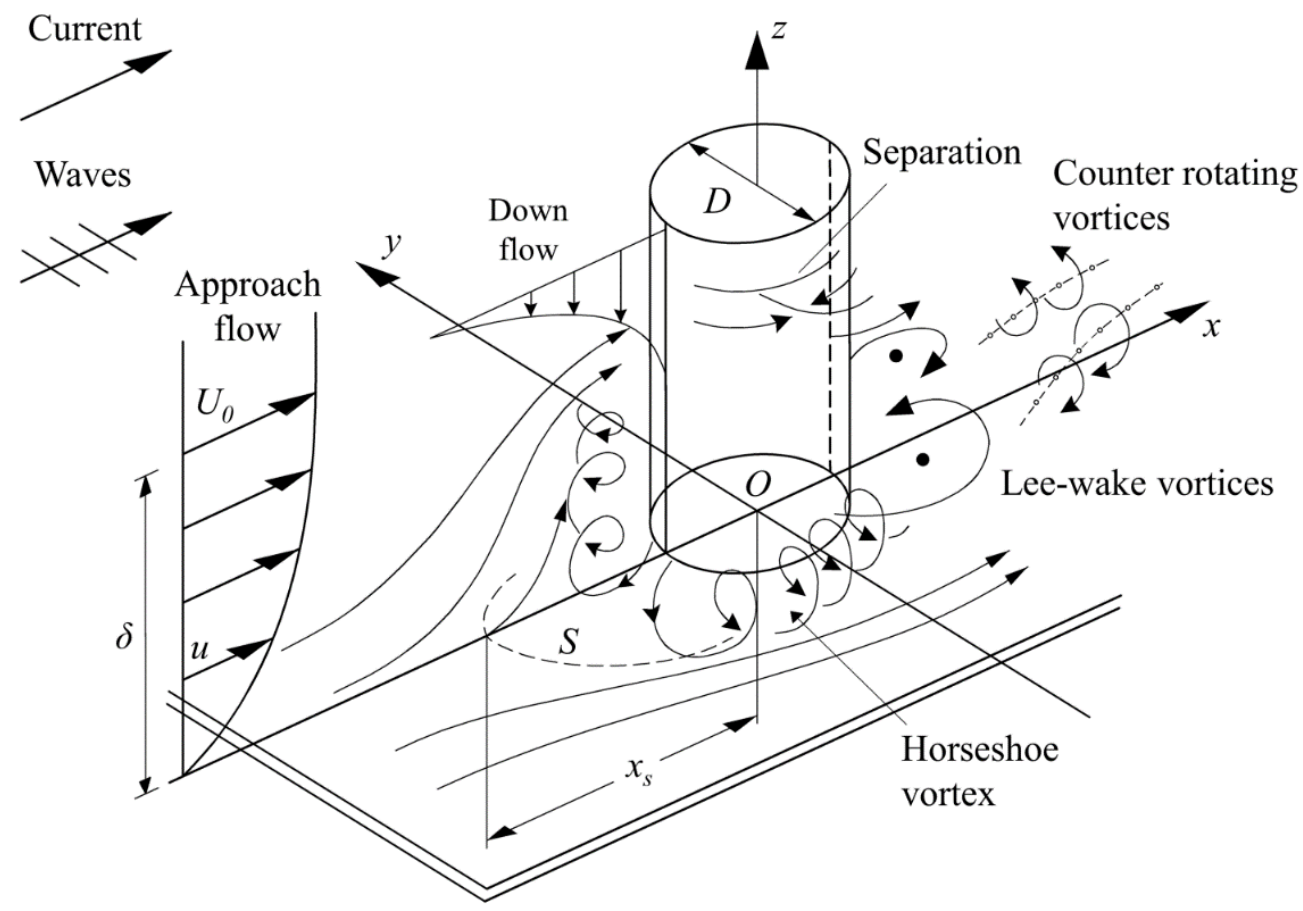

Figure 1. Definition sketch. $S$ : separation line. $\delta$ is the boundary layer thickness. $x_{s}$ is the length characterizing the size of the horseshoe vortex.

Numerical simulations of scour around vertical piles have been previously investigated by several researchers. Important contributions include Olsen and Melaaen (1993); Olsen and Kjellesvig, (1998); Roulund et al. (2005); Gothel and Zilke, (2006); Liu and Garcia, (2008); Zhao and Cheng, (2008); Gothel, (2008); Zhao et al. (2010); Escauriaza and Sotiropoulos (2011a, 2011b); Baranya et al., (2012); Stahlmann and Schlurmann, (2012); Stahlmann, (2014); and Jacobsen et al. (2014b).

The present study is a continuation of the work of Roulund et al. (2005), who studied - both numerically and experimentally - flow and scour around a circular pile exposed to a steady current. The numerical work of Roulund et al. (2005) has, in the present study, been extended to cover the following two cases; (1) scour around a pile exposed to waves, and (2) backfilling of an initially scoured bed around the pile, again, exposed to waves. Two cases are both academically 
and practically important. As for the wave scour, the questions that remain unsettled are, among others: (1) Do the flow features, essential for the scour process, such as the horseshoe vortex and the lee-wake flow, experience significant changes when the flow environment changes from current to waves? (2) Can these changes be captured with the present-day numerical modelling practice? (3) What is the mechanism of scour in the case of waves, and can it be revealed by means of numerical simulations? Likewise, with respect to the backfilling of scour holes by waves: (1) What is the principal mechanism behind the backfilling process? (2) Can the backfilling be captured by the same morphological numerical model developed for scour? (3) Previous applications of numerical scour models have proven successful in predicting the scour properties (such as the scour depth and the time scale). Can these models prove equally successful in predicting the backfilling properties? (4) There is a long debate in the offshore community as to whether the equilibrium depth of scour hole is the same for both the scour process and the backfilling process. Although this latter question has been settled by the experimental findings of Sumer et al. (2013a), can this be confirmed by numerical simulations of the backfilling processes?

This study basically addresses these questions. The present numerical simulations reveal the following features, which deserve early mention. The flow structures such as the horseshoe vortex and the lee-wake flow undergo substantial changes when the flow changes from current to waves. The horseshoe vortex emerges only during a certain portion of the half period of the wave with a significantly diminished "strength", and the lee-wake flow pattern changes from the familiar vortex shedding to multiple vortex shedding regimes governed by the KeuleganCarpenter number. The present numerical simulations reveal (both qualitatively and quantitatively) the experimental observations (see, e.g., Sumer and Fredsøe, 2002) that the leewake vortex flow is the key element behind the mechanism of scour in waves, in contrast to the steady current situation. The present numerical simulations also reveal that the equilibrium depth of scour hole is the same for both the scour process and the backfilling process, confirming numerically, for the first time, the earlier experimental findings of Sumer et al. (2013a).

Earlier results of this study have been presented at the 34th Int. Conf. on Coastal Engineering (Baykal et al., 2014). It may also be noted that the authors have, in a previous study (Baykal et 
al., 2015), investigated the influence of vortex shedding and that of suspended load on scour around a circular pile exposed to a steady current, using the same numerical model as that described in the present paper.

In this study, three kinds of numerical simulations are carried out: (1) rigid bed simulations to investigate the flow features (which will also serve to validate the model); (2) sediment-bed scour simulations; and (3) sediment-bed backfilling simulations. In the rigid bed simulations, the bottom of the calculation domain is kept rigid by turning off the morphological model, whereas, in both scour and backfilling simulations, the morphological model is turned on, and therefore the bed is continuously updated.

The paper is organized as follows. The hydrodynamic and turbulence models used are described in $\S 2$, and the sediment transport and morphological models are described in $\S 3$. The numerical simulations regarding the flow features around a vertical circular pile placed on a rigid bed under steady or oscillatory flow conditions are described and discussed in $\S 4$, while the results of the scour and backfilling simulations are presented in $\$ 5$ and $\S 6$ respectively. The predicted time scales of scour and backfilling from numerical simulations are presented and discussed in $§ 7$. Finally, conclusions are drawn in $\S 8$.

\section{Hydrodynamic model}

\subsection{Governing equations}

The numerical model solves the incompressible Reynolds-averaged Navier-Stokes (RANS) equations

$$
\frac{\partial u_{i}}{\partial t}+u_{j} \frac{\partial u_{i}}{\partial x_{j}}=-\frac{1}{\rho} \frac{\partial p}{\partial x_{i}}+\frac{\partial}{\partial x_{j}}\left[2 v S_{i j}+\frac{\tau_{i j}}{\rho}\right]
$$

combined with the continuity equation

$$
\frac{\partial u_{i}}{\partial x_{i}}=0 .
$$

Here, $S_{i j}$ is the mean-strain-rate tensor 


$$
S_{i j}=\frac{1}{2}\left(\frac{\partial u_{i}}{\partial x_{j}}+\frac{\partial u_{j}}{\partial x_{i}}\right),
$$

where $u_{i}$ are the mean velocities (phase-resolved in the case of waves), $x_{i}$ are the Cartesian coordinates, $t$ is time, $p$ is the pressure, $v$ is the fluid kinematic viscosity, $\rho$ is the fluid density, and $\tau_{i j}$ is the Reynolds stress tensor. The Reynolds stress tensor is defined according to the following constitutive relation:

$$
\begin{gathered}
\frac{\tau_{i j}}{\rho}=-\overline{u_{i}^{\prime} u_{j}^{\prime}}=2 v_{T} S_{i j}-\frac{2}{3} k \delta_{i j}, \\
k=\frac{1}{2} \overline{u_{i}^{\prime} u_{i}^{\prime}},
\end{gathered}
$$

in which $\delta_{i j}$ is the Kronecker delta, $v_{T}$ is the eddy viscosity, and $k$ is the turbulent kinetic energy density, and the overbar denotes ensemble averaging.

The turbulence closure is achieved by the two-equation $k$ - $\omega$ turbulence model of Wilcox (2006, 2008). Based on the authors' experience, the present model behaves similarly to other $k-\omega$ variants, e.g. the $k-\omega$ SST models used in our previous studies of Roulund et al. (2005) and Dixen et al. (2013), while requiring fewer closure coefficients. Wilcox (2006) further discusses the new version of the $k-\omega$, the version used in the present study, and earlier versions created by Wilcox et al., see Wilcox (2006, p. 127 and p. 152).

In the present model the eddy viscosity is defined by

$$
v_{T}=\frac{k}{\widetilde{\omega}}, \quad \tilde{\omega}=\max \left\{\omega, C_{\lim } \sqrt{\frac{2 S_{i j} S_{i j}}{\beta^{*}}}\right\},
$$

which incorporates a stress limiting feature, with $C_{l i m}=7 / 8$. The model equations for the quantities $k$ and $\omega$ read as follows

$$
\begin{gathered}
\frac{\partial k}{\partial t}+u_{j} \frac{\partial k}{\partial x_{j}}=\frac{\tau_{i j}}{\rho} \frac{\partial u_{i}}{\partial x_{j}}-\beta^{*} k \omega+\frac{\partial}{\partial x_{j}}\left[\left(v+\sigma^{*} \frac{k}{\omega}\right) \frac{\partial k}{\partial x_{j}}\right], \\
\frac{\partial \omega}{\partial t}+u_{j} \frac{\partial \omega}{\partial x_{j}}=\alpha \frac{\omega}{k} \frac{\tau_{i j}}{\rho} \frac{\partial u_{i}}{\partial x_{j}}-\beta \omega^{2}+\frac{\sigma_{d}}{\omega} \frac{\partial k}{\partial x_{j}} \frac{\partial \omega}{\partial x_{j}}+\frac{\partial}{\partial x_{j}}\left[\left(v+\sigma \frac{k}{\omega}\right) \frac{\partial \omega}{\partial x_{j}}\right],
\end{gathered}
$$

in which $\omega$ is the specific dissipation rate defined by 


$$
\omega=\frac{\varepsilon}{k \beta^{*}}
$$

with $\varepsilon$ being the dissipation of turbulent kinetic energy

$$
\varepsilon=v \overline{\frac{\partial u_{i}^{\prime}}{\partial x_{k}} \frac{\partial u_{i}^{\prime}}{\partial x_{k}}}
$$

The quantity $\sigma_{d}$ in Eq. 8 is

$$
\sigma_{d}=H\left\{\frac{\partial k}{\partial x_{j}} \frac{\partial \omega}{\partial x_{j}}\right\} \sigma_{d 0}
$$

in which $\mathrm{H}\{\cdot\}$ is the Heaviside step function, taking a value of zero when the argument is negative, and a value of unity otherwise. The standard model closure coefficients are used: $\alpha=13 / 25, \beta=\beta_{0} f_{\beta}, \beta_{0}=0.0708, \beta^{*}=9 / 100, \sigma=1 / 2, \sigma^{*}=3 / 5, \sigma_{d 0}=1 / 8$ and with

$$
f_{\beta}=\frac{1+85 \chi_{\omega}}{1+100 \chi_{\omega}}, \quad \chi_{\omega} \equiv\left|\frac{\Omega_{i j} \Omega_{j k} S_{k i}}{\left(\beta^{*} \omega\right)^{3}}\right|, \quad \Omega_{i j} \equiv \frac{1}{2}\left(\frac{\partial u_{i}}{\partial x_{j}}-\frac{\partial u_{j}}{\partial x_{i}}\right) .
$$

The turbulence model does not take into account so-called "high concentration" effects such as hindered settling of grains or e.g. turbulence damping due to density gradients in the water-sand mixture. This is justified, as these effects are of primary significance only on fine sand beds exposed to high Shields parameters (e.g. Fuhrman et al. 2013).

\subsection{Boundary conditions}

The hydrodynamic model described above is subject to the following boundary conditions. The top boundary of the computational domain (Figure 2) is treated as a frictionless slip wall, with horizontal velocities and scalar hydrodynamic quantities having wall-normal zero gradient $(\partial / \partial n=0)$, Neumann conditions. It is therefore important to emphasize that, in the case of the present wave simulations, the top boundary does not represent the free surface of real waves, the flow beneath which will be approximated by an oscillatory flow, as will be described in the following paragraphs. The application of a "lid" at the top boundary rather than a free surface facility is a commonly used and valid approach in numerical simulations of flow and scour around structures, Liang and Cheng (2005), Fuhrman et al. (2104). It may be noted that the lid at the top boundary is not rigid, and finite velocities were allowed perpendicular to the upper 
boundary in our previous study, Baykal et al. (2015). However, this boundary condition has been replaced in the simulations reported in this paper by the zero-wall-normal-velocity condition.

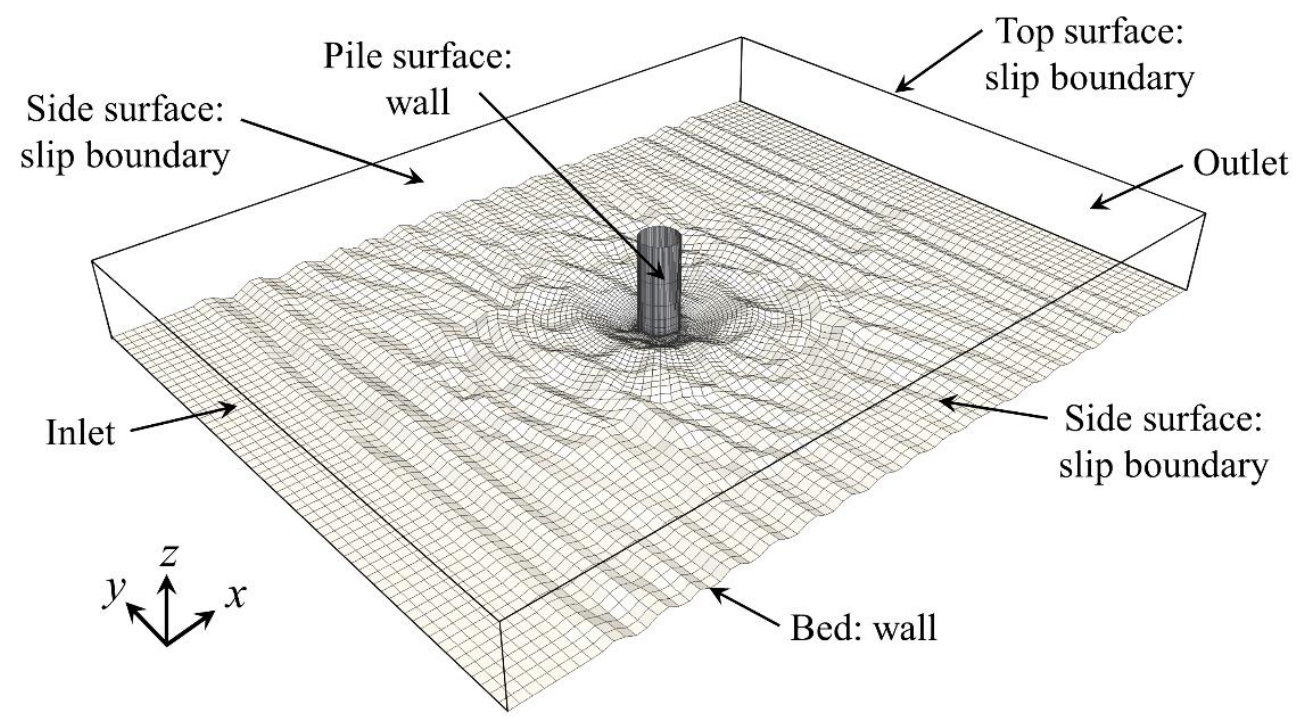

Figure 2. Computational mesh and boundaries.

At the sides of the computational domain, Figure 2, zero-gradient conditions $(\partial / \partial n=0)$, Neumann conditions, are applied for all quantities except for the three components of the velocity, where slip boundary conditions are applied to these.

At friction wall boundaries (i.e. at the bottom and the pile surface), a no-slip condition is imposed whereby velocities are set to zero and a generalized wall function approach developed by Fuhrman et al. (2014) is used. With this approach, the friction velocity $U_{f}$ is determined from the tangential velocity at the nearest cell center, using the Cebeci and Chang (1978) velocity profile which is a generalized version of the van Driest (1956) profile extended to incorporate surface roughness. Further details of the approach can be found in Fuhrman et al. (2014) and Baykal et al. (2015). Nikuradse's equivalent sand roughness of the bed surface is taken as $k_{s}=2.5 d$ in which $\mathrm{d}$ is the grain size. The surface roughness of the pile is kept sufficiently small $\left(k_{s}=1 \times 10^{-5} \mathrm{~m}\right)$ such that it is effectively modelled as hydraulically smooth. 
The boundary conditions of $u, k$ and $\omega$ at the inlet for current flow conditions is obtained from a preliminary hydrodynamic simulation for the domain without the pile, where the flow is driven by a constant horizontal pressure gradient based on the desired undisturbed friction velocity, $U_{f}$ $=[-(h / \rho) \times(\partial p / \partial x)]^{0.5}$ (see also Baykal et al., 2015). As the steady-state flow condition is reached for the steady current, the $u, k$ and $\omega$ profiles at the position of cylinder are used as the inlet boundary conditions in the actual simulation for the same calculation domain with the cylinder in place.

Regarding the wave case, to drive the desired wave conditions, as in Fuhrman et al. (2014), the following boundary conditions, are imposed only at the left-hand (inlet) boundary (see also Liang and Cheng, 2005):

$$
\begin{gathered}
u=U_{m} \sin \left(\frac{2 \pi}{T_{w}} t\right), \quad v=0, \\
k=k_{m}\left[\sin \left(\frac{2 \pi}{T_{w}} t\right)\right]^{2}, \quad k_{m}=0.0005 U_{m}^{2}, \\
\omega=\omega_{m}\left|\sin \left(\frac{2 \pi}{T_{w}} t\right)\right|, \quad \omega_{m}=\frac{k_{m}}{100 v},
\end{gathered}
$$

in which $U_{m}$ is the maximum free stream velocity, and $T_{w}$ is the wave period. The inlet boundary conditions does not move from one side of the computational domain to the other side during each wave period.

For both current and wave cases, at the inlet for the remaining quantities (concentration, $c$, and pressure, $p$ ), Neumann boundary conditions are applied. At the opposite right-hand boundary (outlet), zero-gradient boundary conditions are imposed for all quantities except the pressure term, which is taken as zero at the outlet boundary. For each wave case considered, the initially still domain is introduced to the specific wave climate via the left-hand boundary for total warmup duration of $10 T_{w}$. During this warm-up period hydrodynamic and sediment transport fields are allowed to develop, with bed morphology switched off, so that the bed remains still even if the sediment transport computations are carried out based on the hydrodynamic computations (Fuhrman et al., 2014). At the end of this warm-up period, denoted as $t=0$, the morphological model is activated, and the bed is allowed to evolve freely (Fuhrman et al., 2014). 


\section{Sediment transport and morphological models}

This section describes the sediment transport and morphological models used in the study. The implementation of these models, including a detailed account of numerical aspects, has been described by Jacobsen (2011) and Jacobsen et al. (2014a). The description of the models used in this study is given previously in Baykal et al. (2015), and therefore, in what follows the description will be presented only briefly.

\subsection{Sediment transport model}

The model makes use of separate bed and suspended sediment load descriptions. The rate of bed load sediment transport, $q_{b}$, is calculated based on the method adopted by Roulund et al. (2005), who generalized the sediment transport formulation of Engelund and Fredsøe $(1976,1982)$ to account for three-dimensional effects as well as bed-slope modifications. A detailed account of this method has been given in Roulund et al. (2005), and the reader is referred to the latter publication.

The suspended load description is, on the other hand, based on a turbulent diffusion equation of the form

$$
\frac{\partial c}{\partial t}+\left(u_{j}-w_{s} \delta_{j 3}\right) \frac{\partial c}{\partial x_{j}}=\frac{\partial}{\partial x_{j}}\left[\left(v+v_{T}\right) \frac{\partial c}{\partial x_{j}}\right],
$$

in which $c$ is the suspended sediment concentration, and $w_{s}$ is the settling velocity of sediment grains. We note that the kinematic viscosity of fluid, $v$, is maintained here for numerical reasons, discussed in Jacobsen et al. (2014a).

Eq.16 is solved on a truncated version of main computational mesh used for the hydrodynamic computations. In the truncated mesh, the near-bed cells below the reference level $b$ are removed. Therefore, Eq. 16 is only solved starting from the reference level and above. Below this level sediment transport is considered as bed load and therefore not modelled by Eq. 16 (see Jacobsen et al., 2014a, e.g. their Fig.2, for further details). 
At the top and at the structure boundaries, a zero-flux condition for concentration is utilized, and for the other boundaries Neumann conditions are applied.

At the bottom boundary of the truncated mesh, using method of Engelund and Fredsøe (1976), the concentration, $c$, at the reference level $z=b$ is set to

$$
c_{b}(\theta)=\frac{c_{0}}{\left(1+1 / \lambda_{b}\right)^{3}},
$$

where $c_{0}=0.6$, and the linear concentration $\lambda_{b}$ is

$$
\lambda_{b}^{2}=\frac{\kappa^{2} \alpha_{1}^{2}}{0.013 s \theta}\left(\theta-\theta_{c}-\frac{\pi}{6} \mu_{d} p_{E F}\right),
$$

where $\kappa=0.4$ is the von Karman constant, and

$$
p_{E F}=\left[1+\left(\frac{\pi \mu_{d}}{6\left(\theta-\theta_{c}\right)}\right)^{4}\right]^{-1 / 4},
$$

is the probability of moving grains, and

$$
\theta=\frac{\tau_{0}}{\rho g(s-1) d}=\frac{U_{f}^{2}}{g(s-1) d},
$$

is the Shields parameter, in which $\tau_{0}=\rho U_{f}^{2}$ is the wall shear stress, $s=\rho_{s} / \rho$ is the specific gravity of the sediment grains taken as 2.65, $\rho_{s}$ is the density of sand grains, $g$ is the gravitational acceleration and $d$ is the grain size. In the computations, the critical value of the Shields parameter corresponding to the initiation of sediment motion on a horizontal bed, $\theta_{c}$, is 0.05 . The bed-slope effect is accounted for as in Roulund et al. (2005). In the case of waves, $U_{f}$ in the above equation is the phase-resolved instantaneous friction velocity. Throughout the present work, the coefficient of dynamic friction is set to $\mu_{d}=0.51$ (Fredsøe and Deigaard,1992, p.218) and the reference level is taken as $b=\alpha_{1} d=3.5 d$ (Fuhrman et al.,2014; Baykal et al. 2014; 2015).

To prevent un-physical 'overloading' conditions (i.e. where reference concentration $c_{b}$ is forced to be smaller than the concentration immediately above) from occurring in the model, the solution suggested by Justesen et al. (1986) is finally invoked. That is, if the concentration close 
to the bed exceeds the reference concentration $c_{b}(\theta)$, the value in practice is taken from the cell adjacent to the boundary.

Validation of the present suspended-sediment-transport model has been achieved in Jacobsen (2011) in terms of the relative concentration $c / c_{b}$ for various fundamental cases (without structure) such as equilibrium suspended sediment transport in currents (Vanoni distribution, Vanoni, 1975, Fig.34, and van Rijn distribution, van Rijn, 1984), and non-equilibrium suspended sediment transport, again, in currents (Hjelmfelt and Lenau, 1970). Likewise, the model has also been validated for sediment concentrations under breaking waves in Jacobsen (2011). It is to be noted, however, that absolute values of sediment concentrations are expected to be influenced by the change of turbulence properties (such as von Karman constant) due to suppression of turbulence by the presence of sediment in suspension, particularly in cases with very high sediment concentrations. It is apparent that the influence of sediment on turbulence, and therefore on sediment concentrations (and hence on scour) is worth exploring in future studies, as already noted in Section 2.1.

\subsection{Morphological model}

Morphology of the bed elevation $h_{b}$ is based on the sediment continuity (Exner) equation

$$
\frac{\partial h_{b}}{\partial t}=-\frac{1}{1-n}\left[\nabla \cdot \mathbf{q}_{\mathbf{b}}+E_{s}+D_{s}\right]
$$

in which the morphological change is split into contributions from the bed load transport $\mathbf{q}_{\mathbf{b}}$ and the suspended load transport $\left(E_{s}\right.$ and $\left.D_{s}\right)$. Here, $E_{s}$ and $D_{s}$ are the erosion and deposition stemming from suspended load, respectively, $n=0.4$ is the porosity of the sediment bed. As given by Jacobsen et al. (2014a), the evaluation of bed load contribution $\left(\nabla \cdot \mathbf{q}_{\mathbf{b}}\right)$ in the model yields a change in the normal direction to the bed, however, morphological updating is along the vertical axis, $\mathbf{e}_{g}$, hence a projection is needed. The bed level change due to bed load, $\Delta h_{b, b}$, is then given as

$$
\Delta h_{b, b}=-\frac{1}{1-n} \frac{\nabla \cdot \mathbf{q}_{b}}{\left|\mathbf{n} \cdot \mathbf{e}_{g}\right|} \Delta t,
$$


where $\mathbf{n}$ is the bottom face unit normal. The area of the face is $\|\mathbf{N}\|_{2}$, where $N=\|\mathbf{N}\|_{2} \mathbf{n}$. The bed level change due to erosion and deposition, $\Delta h_{b, s}$, is then given as

$$
\Delta h_{b, s}\left|\mathbf{e}_{g} \cdot \mathbf{N}\right|=\frac{1}{1-n}\left(D_{v}-E_{v}\right) \Delta t
$$

where $D_{v}$ and $E_{v}$ are given as:

$$
E_{v}=\left(v+v_{T}\right) \frac{\partial c}{\partial n}\|\mathbf{N}\|_{2}=\left(v+v_{T}\right) \mathbf{N} \cdot \nabla c, \quad D_{v}=\left|\left(\mathbf{w}_{s}+\mathbf{u}\right) \cdot \mathbf{N}\right| c_{b} .
$$

Above, $n$ is the local coordinate normal to the bottom boundary face. Here, the contribution due to deposition $D_{v}$ only comes from the projection of $\mathbf{w}_{s}+\mathbf{u}$ onto the face normal vector, whereas the tangential projection, $\mathbf{w}_{s, \tau}\left(=\left\|\mathbf{n} \times\left(\left(\mathbf{w}_{\mathrm{s}}+\mathbf{u}\right) \times \mathbf{n}\right)\right\|_{2}\right)$, does not contribute.

Above, $\mathbf{w}_{s}$ is the sediment fall velocity determined using drag coefficient for natural sand, see Fredsøe and Deigaard (1992, pp.198-199). For more specific details regarding the numerical evaluation of the three terms on the right-hand-side of Eq. 21, the reader is referred to Jacobsen et al. (2014a). It is to be noted that, in the morphology calculations, the time steps are taken exactly the same as in the hydrodynamic calculations, i.e., the morphological model does not make use of morphological rates averaged over any larger time scales or morphological acceleration. The reason for this choice is the rapid change in the development of the scour hole in the initial stages of the scour process that requires a close coupling between the hydrodynamics and the bed evolution, because the rate of bed level change is similar in magnitude to the fall velocity of the sediment. Since the present modelling efforts are restricted to one morphological updating routine, the one-to-one coupling is favoured to capture the initial processes most accurately. Moreover, this strategy limits the deformation in the mesh that will occur over a single time step, and is hence effective in ensuring that the resulting bed motions do not exceed the near-bed cell size (typically the order of the grain size). At later stages of scour development, it may be justified to average the transport properties over e.g. one wave period (see e.g. Jacobsen et al. 2014b) and use this information in the updating, though such a change in morphological updating approach has not been utilized in the present work. A change in the morphological updating routine during a simulation should be considered carefully, as the change can affect sediment mass conservation, see the analysis in Jacobsen (2015). 
It is finally noted that, to prevent excessive erosion induced by the imposed uniform flow at the boundary, the sea bed is fixed at the left (inlet) boundary for the steady current case and at both boundaries (inlet and outlet) for the wave cases, and relaxed to full morphology over a distance spanning a few pile diameters $(1.5 D$ from inlet for steady current, and $2 D$ from both inlet and outlet for waves).

Experience has shown that, if left un-checked, the morphological model can lead to local bed slopes in excess of the angle of repose. To avoid this, the sand slide model described in detail by Roulund et al. (2005) is implemented. In the present work, this sand slide model is activated at positions where the local bed angle exceeds the angle of repose $\varphi_{s}=32^{\circ}$, and is de-activated once the local bed angle is reduced to $30^{\circ}$. Additionally, some local filtering of the bed was necessary in the simulations for stability reasons. For this purpose, a filtering algorithm based on the leastsquares method is applied to the morphological rates in the near vicinity of the pile to smooth out small-sized, high-wavenumber, unphysical ripples that can occur inside the scour hole adjacent to the pile, where the numerical stability of the mesh motion computations becomes critical due to increased rates of change in bed elevations. (Note that observations show that these highwavenumber, small-size ripples in the scour hole in the live-bed scour regime are unphysical, Sumer et al., 1992, and Roulund et al., 2005, among others.) The strength of the filter inside and around the scour hole is kept variable with the use of a hyperbolic function based on the radial distance from the origin of the domain. The filter is weak outside the scour hole and the far-field bed ripples are resolved but the very small-sized, unphysical ripples inside the scour hole are filtered, since the filter is strongest next to the cylinder. The mesh motion routine is based on the interpolation of the rate of bed level change from the faces to the vertices on the bottom and solution of a Laplace equation for the motion of the mesh vertices as described in Jasak and Tuković (2006). The mesh is updated each time the bed is updated. The method applied in this work has a slight mass conservation error as shown in Jacobsen (2015). This mass conservation error was estimated from the numerical results and it was found neither to impact the results qualitatively nor quantitatively.

The equations comprising the fully-coupled model outlined above are solved numerically using the open-source Computational Fluid Dynamics (CFD) toolbox OpenFOAM ${ }^{\circledR}$ (version 1.6-ext, 
2010), making use of a finite volume spatial discretization with a collocated variable arrangement, in conjunction with PIMPLE algorithm, which is a combination of PISO (Pressure Implicit with Splitting of Operators; Issa, 1986) and SIMPLE (Semi-Implicit Method for Pressure-Linked Equations; Patankar and Spalding, 1972) algorithms for transient incompressible flows of Newtonian fluids, provided by OpenFOAM ${ }^{\circledR}(2010)$.

\subsection{Computational mesh}

The computational domain is discretized into finite volumes of quadrilateral blocks in varying shapes and dimensions. During the computations, the mesh is continuously updated to adjust to the changes of bed topography. The computational domain used in the simulations has the following dimensions: Length, $l=20 D$, Width, $w=15 D$, and Height, $h=2 D$ where $D$ is the pile diameter. The total number of cells is $\mathrm{O}\left(10^{5}\right)$ in the simulations. The pile is located at the center of the domain $(x=0$ and $y=0)$. Table 1 summarizes the mesh characteristics for different calculation types.

Table 1. Characteristics of meshes used in the numerical simulations.

\begin{tabular}{lccc}
\hline Calculation type & $\begin{array}{c}\text { Current rigid } \\
\text { bed or live- } \\
\text { bed scour }\end{array}$ & $\begin{array}{c}\text { Wave rigid } \\
\text { bed or live- } \\
\text { bed scour }\end{array}$ & $\begin{array}{c}\text { Wave live- } \\
\text { bed } \\
\text { backfilling }\end{array}$ \\
\hline Total number of cells & 94080 & 119808 & 104448 \\
\hline Number of cells across the water depth & 14 & 16 & 16 \\
\hline $\begin{array}{l}\text { Minimum cell thickness in vertical z-direction at the } \\
\text { bottom of computational domain }\end{array}$ & $0.010 D$ & $0.011 D$ & $0.015 D$ \\
\hline Number of cells around the pile perimeter & \multicolumn{4}{c}{96} \\
\hline Minimum cell (radial) thickness next to cylinder & $0.020 D$ & $0.020 D$ & $0.025 D$ \\
\hline
\end{tabular}

As an indication of the computational expense, a fully coupled hydrodynamic and morphological calculation lasting 1 min of physical time for the three-dimensional scour around a vertical pile subjected to steady current requires approximately 10 days of CPU time, when simulated in parallel on eight modern processors. We also note that, for the same computational mesh and using the same computational power, the hydrodynamic calculations for 1 min of physical time last approximately 1.5 days. The duration for fully coupled hydrodynamic and morphological calculation lasting 1 min of physical time for the three-dimensional scour around a vertical pile subjected to waves requires approximately 3-4 days. The total physical time covered in the 
simulations presented here ranges between 7.5 min to 1 hour, resulting in computation times up to 8 months (especially for backfilling cases). In all the calculations, the time step is kept as variable to ensure that certain Courant numbers selected separately for the numerical stability in each of hydrodynamic, suspended sediment and morphological calculations are not exceeded. Throughout the study, it has been seen that the morphological calculations have essentially "controlled" the speed of the simulations. We further note that considerable effort has been put into optimizing the computational mesh for the best grid and time convergence. Finer computational meshes could not be used as CPU times increased significantly due to numerical stability considerations. Simulation of similar problems at larger scales within reasonable computational times requires further improvements, mainly in the mesh motion routines and parallelism of the present model. Moreover, the morphological time step might be increased compared to the hydrodynamic time step as in Stahlmann, and Schlurmann (2012) and Stahlmann (2014).

\section{Rigid-bed simulations}

In the rigid-bed simulation tests, the flow features around a vertical circular pile mounted on a horizontal, rigid, plane bed and subjected to a steady current or oscillatory flow (Figure 1) are investigated through numerical simulations for the test conditions given in Table 2. The steady current test condition is based on Test 1 of Sumer et al.'s (2013a) current experiment in which a $40 \mathrm{~mm}$ diameter circular pile was exposed to a steady current with an initially plane sediment bed. The test wave conditions are, on the other hand, based on the experiments of Sumer et al.'s (1997) regular wave experiments in a wave flume with a rigid, plane bed, with (1) Test 8, corresponding to a Keulegan-Carpenter number of $K C=10$, and (2) Test 14, corresponding to $K C=20$, in which $K C$ is the Keulegan-Carpenter number defined by

$$
K C=\frac{U_{m} T_{w}}{D},
$$

in which $U_{m}$ is the maximum value of the horizontal component of the orbital velocity at the bed in the Sumer et al. (1997) experiment, or the maximum value of the oscillatory flow in the numerical simulations, 


$$
U(\omega t)=U_{m} \sin (\omega t)
$$

with $\omega$ being the angular frequency of the oscillatory flow, $\omega=2 \pi / T_{w}$ and $T_{w}$ is the period. The pile diameter in Sumer et al.'s (1997) wave experiments is also $D=40 \mathrm{~mm}$.

The boundary layer thickness $\delta$ in Table 2 is taken as the flow depth in Test 1 of Sumer et al. (2013a) while that in Tests 8 and 14 of Sumer et al. (1997) is taken as the distance across the boundary layer from the wall to a point where the flow velocity essentially reaches the maximum value of the free stream velocity in both experiments and the simulations, according to the definition given in Jensen et al. (1989, Fig. 24).

Table 2. The test conditions for rigid bed simulations. All cases use pile diameter $D=40 \mathrm{~mm}$.

\begin{tabular}{|c|c|c|c|}
\hline & Steady current & & \\
\hline Test No. of the indicated reference & $\begin{array}{l}\text { Test } 1 \text { of } \\
\text { Sumer et al.'s } \\
\text { (2013a) flume } \\
\text { expts. }\end{array}$ & $\begin{array}{c}\text { Test } 8 \text { of } \\
\text { Sumer et } \\
\text { al.'s (1997) } \\
\text { wave flume } \\
\text { expts. }\end{array}$ & $\begin{array}{c}\text { Test } 14 \text { of } \\
\text { Sumer et } \\
\text { al.'s (1997) } \\
\text { wave flume } \\
\text { expts. }\end{array}$ \\
\hline Domain height, $h(\mathrm{~m})$ & 0.08 & 0.08 & 0.08 \\
\hline Boundary layer thickness, $\delta(\mathrm{m})$ & 0.08 & 0.0024 & 0.0024 \\
\hline Keulegan-Carpenter number, $K C$ & $\infty$ (Current) & 10 & 20 \\
\hline Mean flow velocity, $V(\mathrm{~m} / \mathrm{s})$ & 0.413 & - & - \\
\hline $\begin{array}{l}\text { Maximum value of the undisturbed orbital velocity } \\
\text { at the bed in the experiments, or the maximum } \\
\text { value of the free stream velocity of the oscillatory } \\
\text { flow in the numerical simulations, } U_{m}(\mathrm{~m} / \mathrm{s})\end{array}$ & - & 0.094 & 0.183 \\
\hline Wave period, $T_{w}(\mathrm{~s})$ & - & 4.4 & 4.4 \\
\hline $\begin{array}{l}\text { Boundary-layer-thickness Reynolds number, } \operatorname{Re}_{\delta}= \\
V \delta / v \text { (or } U_{m} \delta / v \text { for waves) }\end{array}$ & $3.3 \times 10^{4}$ & $2.3 \times 10^{2}$ & $2.3 \times 10^{2}$ \\
\hline $\begin{array}{l}\text { Pile-diameter Reynolds number, } R e_{D}=V D / v \text { (or } \\
U_{m} D / v \text { for waves) }\end{array}$ & $1.7 \times 10^{4}$ & $3.8 \times 10^{3}$ & $7.2 \times 10^{3}$ \\
\hline $\begin{array}{l}\text { Friction velocity, } U_{f} \text { or the maximum value of the } \\
\text { friction velocity } U_{f m} \text { for waves in the experiments, } \\
\text { or that for oscillatory flow in the simulations, } \\
(\mathrm{m} / \mathrm{s})\end{array}$ & 0.019 & 0.019 & 0.013 \\
\hline $\begin{array}{l}\text { Nikuradse's equivalent sand roughness of the bed } \\
k_{s}=2.5 d(\mathrm{~m})\end{array}$ & $4.25 \times 10^{4}$ & $4.25 \times 10^{4}$ & $4.25 \times 10^{4}$ \\
\hline $\begin{array}{l}\text { Dimensionless bed roughness } k_{s}^{+}=k_{s} U_{f} / v \text { (or } \\
k_{s} U_{f m} / v \text { for waves in the experiments, or for } \\
\text { oscillatory flow in the simulations) }\end{array}$ & 8.1 & 8.1 & 5.5 \\
\hline
\end{tabular}


The quantity $U_{f}$ in Table 2 is the friction velocity in the case of current defined by

$$
U_{f}=\left(\bar{\tau}_{0 \infty} / \rho\right)^{1 / 2},
$$

in which $\bar{\tau}_{0 \infty}$ is the undisturbed bed shear stress and $\rho$ is the density of water. The quantity $U_{f m}$ in Table 2 is the maximum value of the friction velocity for waves in the experiments, or that for oscillatory flow in the simulations:

$$
U_{f m}=\left(\bar{\tau}_{0 m} / \rho\right)^{1 / 2},
$$

which $\bar{\tau}_{0 m}$ is the maximum value of the bed shear stress.

Sumer et al. (2013a) states that the quantity $U_{f}$ in Table 2 is based on the measured velocity profile using the log-fit exercise, which is later confirmed checking against the values found from the Colebrook-White formula (Schlichting, 1979). The quantity $U_{f m}$ in Table 2 belonging Sumer et al.'s (1997) wave flume experiments is stated to be obtained through bed-shear stress measurements by a two-component hot-film probe. In the numerical model, the friction velocities in are obtained from the computed wall shear stresses.

\subsection{Horseshoe vortex}

The horseshoe vortex (Figure 1) is caused by the combined action of the shear in the approach flow, and the adverse pressure gradient produced by the presence of pile. The separated boundary layer (Figure 1, $S$ being the separation line) subsequently rolls up to form a horseshoe (or necklace)-shaped vortical structure around the pile, which then trails off downstream. From dimensional grounds, the non-dimensional quantities describing the horseshoe vortex around a smooth pile depend on: $\delta / D, \operatorname{Re}_{D}$ (or alternatively $R e_{\delta}$ ) in the case of steady currents, and $\delta / D$, $\operatorname{Re}_{D}$ (or alternatively $R e_{\delta}$ ) and $K C$ in the case of waves, in which $\delta$ is the boundary layer thickness. 
In the wave case, small $K C$ numbers mean that the orbital motion of water particles is small relative to the pile diameter. For very small $K C$ numbers, the horseshoe vortex may not even be formed because the stroke of the fluid motion is not large enough for the approach boundary layer to separate (along $S$, Figure 1). For very large $K C$ numbers, on the other hand, the stroke of the fluid motion is so large that the flow for each half period resembles that in a steady current, and therefore the horseshoe vortex is expected to behave in much the same way as in the case of the steady current (Sumer et al., 1997).

Figure 3 illustrates the vortical structures around the pile, obtained in the steady current simulation (Test 1 , Table 2), represented by $Q$ iso-surfaces (in $1 / \mathrm{s}^{2}$ ). The $Q$ iso-surfaces are obtained, using the so-called $Q$-criterion (Hunt et al., 1988). The $Q$-criterion defines a vortex as a coherent fluid region with positive second invariant $(Q>0)$ of the velocity gradient tensor, $A_{i j}=$ $\partial u_{i} / \partial x_{j}$

$$
Q=\frac{1}{2}\left(\left|\Omega_{i j}\right|-\left|S_{i j}\right|\right)>0
$$

that is the region where the magnitude of vorticity tensor, $\Omega_{i j}$, prevails over the magnitude of strain-rate tensor, $S_{i j} . \Omega_{i j}$ and $S_{i j}$ are given, respectively,

$$
\Omega_{i j}=\frac{1}{2}\left(\frac{\partial u_{i}}{\partial x_{j}}-\frac{\partial u_{j}}{\partial x_{i}}\right), S_{i j}=\frac{1}{2}\left(\frac{\partial u_{i}}{\partial x_{j}}+\frac{\partial u_{j}}{\partial x_{i}}\right) .
$$

The $Q$-criterion also implies that local pressure is lower than surrounding pressure within this coherent fluid region.

The simulation clearly reveals the presence of the horseshoe vortex in front of the pile, and the lee-wake shed vortices at the back. (The lee-wake vortices will be discussed in Section 4.2.) The horseshoe vortex in the steady current case has been investigated numerically in great detail with fine resolution simulations of Roulund et al. (2005), Paik et al., (2007), Kirkil and Constantinescu (2005), Kirkil et al., (2005, 2006, 2008), Escauriaza and Sotiropoulos (2011c) and therefore the reader is referred to these works. The remainder of this section will focus on the horseshoe vortex in the case of waves. 
Figure 4 depicts the coherent flow structures around the pile for the oscillatory flow case for Test 14 in Table 2 with $K C=20$, obtained by the same $Q$-criterion technique. The sequence of the flow pictures in Figure 4 is given for phases $\omega t=60^{\circ}, 90^{\circ}, 120^{\circ}$, and $150^{\circ}$ (see Eq. 26 for the definition of $\omega t ; \omega t=0^{\circ}$ corresponding to the phase of zero-upcrossing of the free-stream velocity $U$.) It is important to note that, in Figure 4a, the lee-wake vortices in front of the pile left from the previous half cycle are removed in the flow visualization so that the horseshoe vortex could be visualized more clearly. The free-stream flow is from left to right.

It is seen that, at $\omega t=60^{\circ}$ (Figure $4 \mathrm{a}$ ), the horseshoe vortex is already visible. For further phase values, it exists with even larger magnitudes (e.g., $\omega t=90^{\circ}$, Figure $4 \mathrm{~b}$ ). The horseshoe vortex remains visible for phase values $\omega t=120^{\circ}$ and $150^{\circ}$, albeit with a decreasing degree of strength until $\omega t$ reaches the value $\omega t=150^{\circ}$. The above findings are in agreement with Sumer et al.'s (1997, Fig. 7) wave-flume study where the horseshoe vortex was made visible by use of hydrogen bubble technique. The lee-wake vortices illustrated in Figure 4 will be discussed in Section 4.2 .
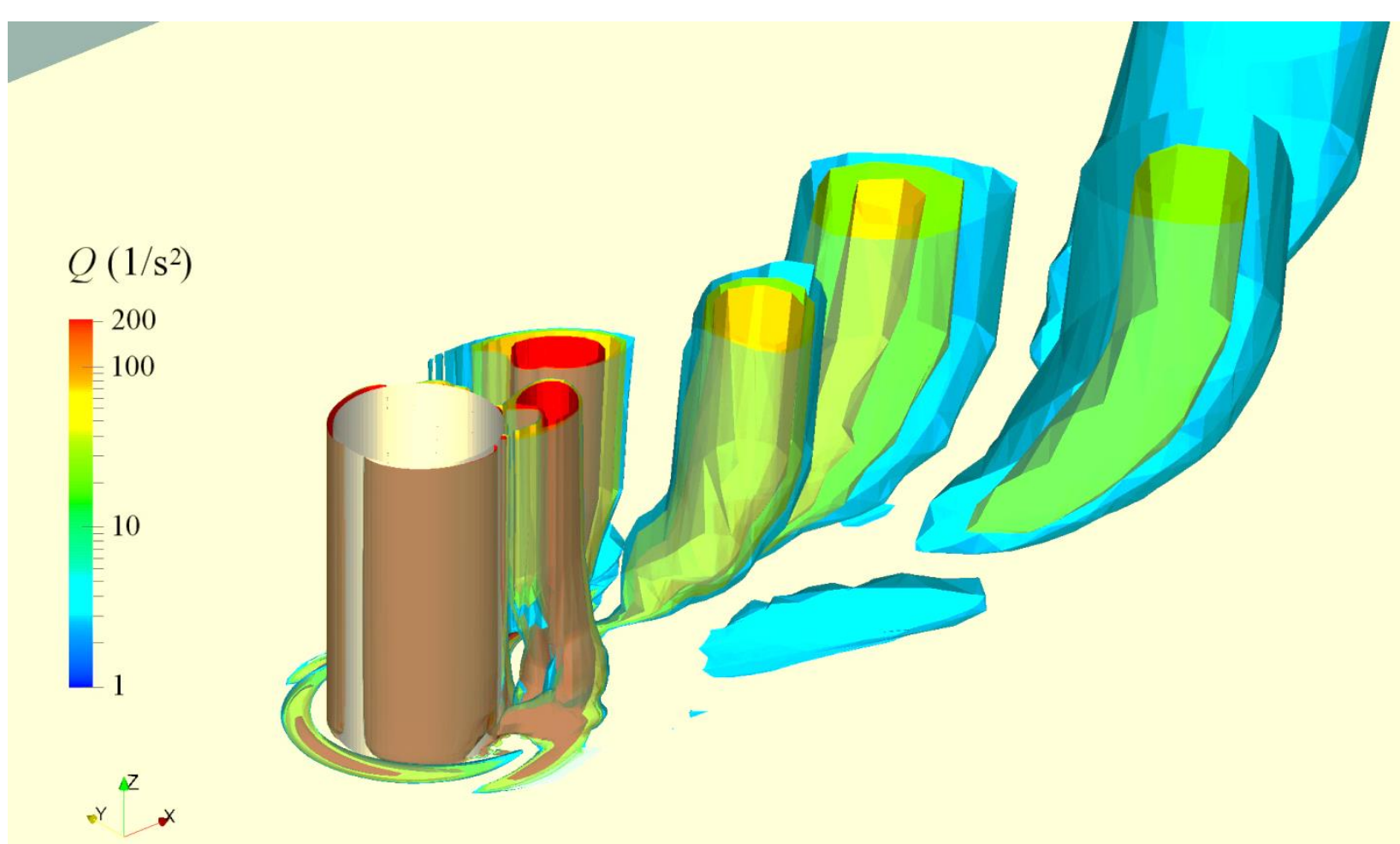
Figure 3. $Q$ representation (in $1 / \mathrm{s}^{2}$ ) for the horseshoe vortex and the shed vortices around the cylinder for steady current, Test 1 of Sumer et al.'s (2013a) flume experiments, given in Table 2.

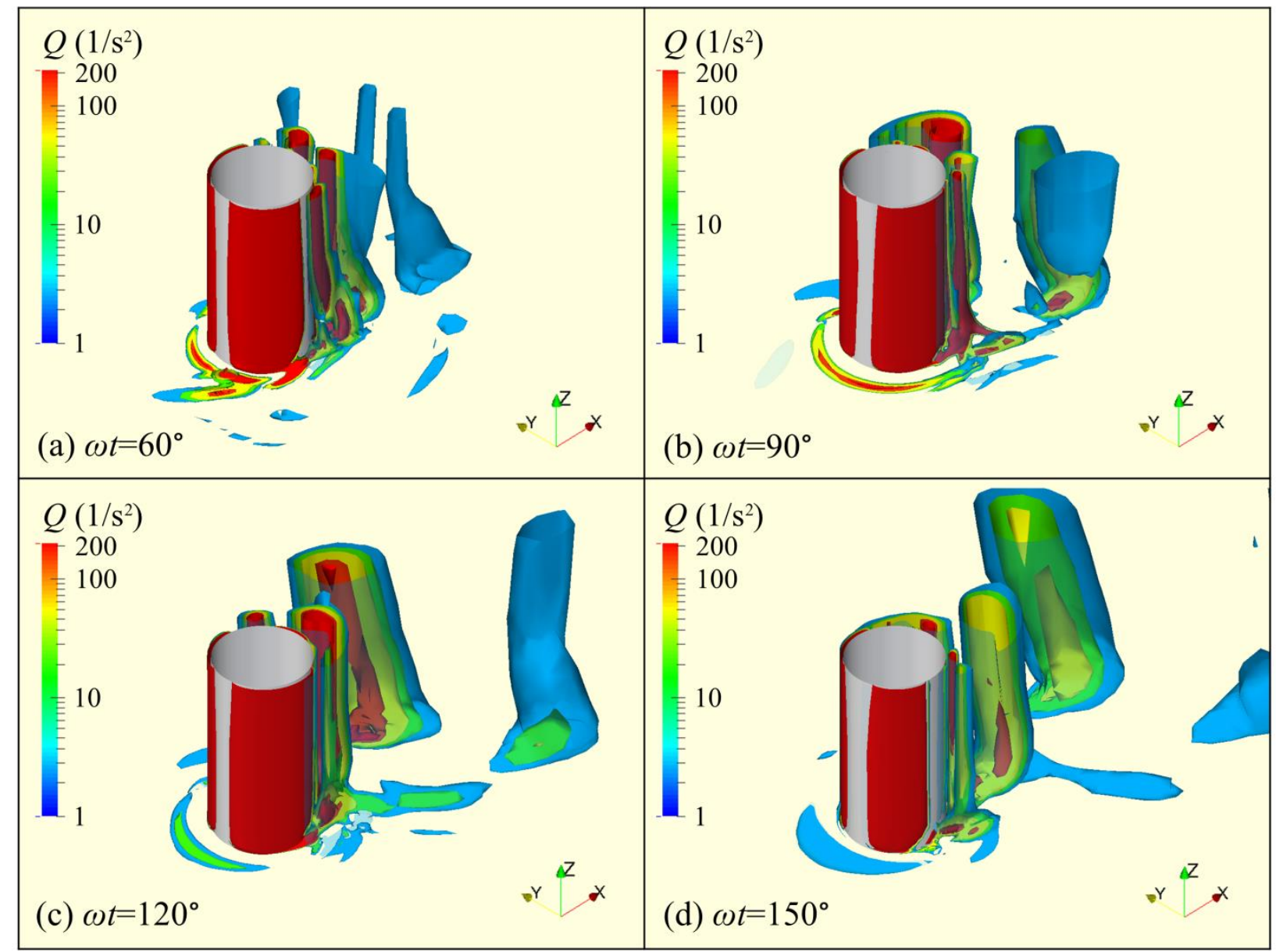

Figure 4. $Q$ representations (in $1 / \mathrm{s}^{2}$ ) for the horseshoe vortex, and the shed vortices around the cylinder at different phases of oscillatory flow of $K C=20$, Test 14 of Sumer et al.'s (1997) wave flume experiments, given in Table 2, at $\omega t=60^{\circ}$ (a), $\omega t=90^{\circ}$ (b), $\omega t=120^{\circ}$ (c), and $\omega t=150^{\circ}$ (d).

Figure 5 depicts the variation of the bed shear stress amplification along the $\mathrm{x}$-axis at the phase values $\omega t=90^{\circ}\left(x / D<0\right.$, the flow is from left to right) and $\omega t=270^{\circ}(x / D>0$, the flow is from right to left). The present results (solid line) are compared with the experimental data of Sumer et al. (1997) (see Fig. 17c \& d on p. 58 and the Test 8 \& 14 data on p. 66 in Sumer et al., 1997) for $K C=10$ and $K C=20$, and the experimental data of Roulund et al. (2005) and Hjorth (1975) (see Fig. 6.18b on p. 133 in Hjorth, 1975; and the Test 1 data in Fig.16 on p.376 in Roulund et al., 2005) for steady current. (It may be noted that the steady current comparison (Figure 5c) was 
presented previously in Baykal et al. (2015), and the results are included here for the sake of completeness.) In the figure, the amplification of the bed shear stress is defined as $\bar{\tau}_{0} / \bar{\tau}_{0 \infty}$ for steady current and $\bar{\tau}_{0} / \bar{\tau}_{0 m}$ for waves, in which $\bar{\tau}_{0}$ is the bed shear stress and $\bar{\tau}_{0 \infty}$ is the undisturbed bed shear stress for steady current and $\bar{\tau}_{0 m}$ is the maximum undisturbed bed shear stress for waves. The negative bed shear stress corresponds to the location of the horseshoe vortex in front of the pile.
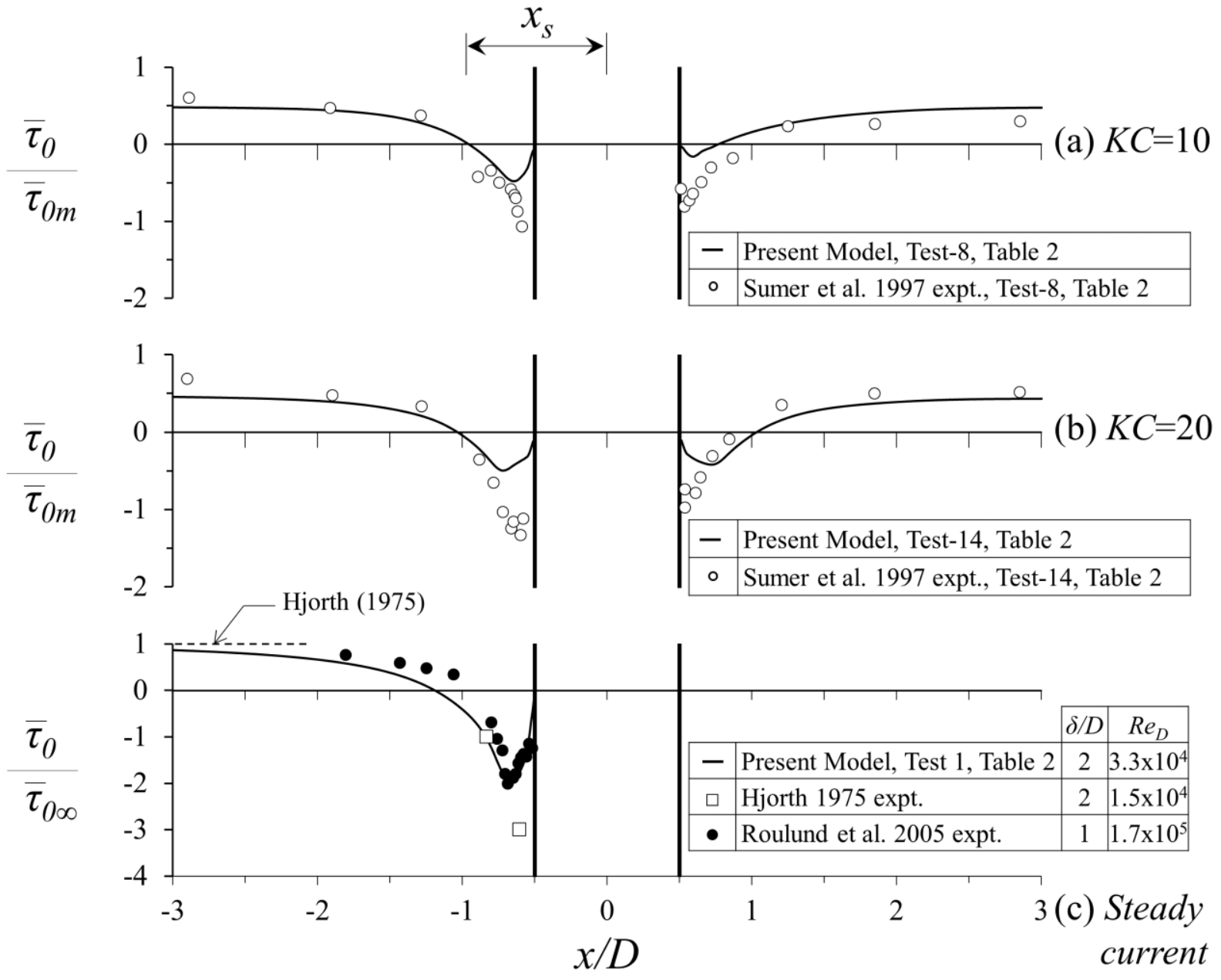

Figure 5. Bed shear stress amplification along the $\mathrm{x}$-direction at the horseshoe vortex side of the pile (at the phase values $\omega t=90^{\circ}$ for $x / D<0$, and $270^{\circ}$ for $x / D>0$ ) for (a) the oscillatory flow of $K C=10$, Sumer et al. 1997 expt. Test 8 in Table 2, (b) $K C=20$, Sumer et al. 1997 expt. Test 14 in Table 2 and (c) steady current, Test 1 in Table 2 (adapted from Baykal et al., 2015). 
Figure 5 shows that, overall, the numerical and experimental results agree well for $|x / D|>0.75$. However, for $0.5<|x / D|<0.75$, the bed shear stress is apparently under-predicted by the numerical model. This discrepancy could be attributed to the under-prediction of horseshoevortex strength, exhibited in Figure 4, and this is linked to the dissipative nature of RANS (the present study) compared to eddy-resolving models such as large-eddy simulation (LES) and detached eddy simulation (DES) (Paik et al., 2007; Kirkil and Constantinescu, 2005; Kirkil et al., 2005; 2006; 2008; Escauriaza and Sotiropoulos, 2011c). It is to be noted that the standard approach to simulate coherent structures (such as the horseshoe vortex) is to use LES simulation. Using a RANS approach, these structures can be significantly damped or smoothed (because of the large eddy viscosity used), as pointed out by one of the reviewers. Whether or not RANS approach is the best way to study horseshoe vortices has not yet been studied in a clear and systematic way. It appears that this aspect of the problem would be worth exploring further. In this context, the authors recognize the LES approach to resolve coherent structures around wallmounted cylinders by Professor Constantinescu's group at the University of Iowa (Kirkil and Constantinescu, 2005; Kirkil et al., 2005; 2006; 2008), and believe that their work may serve as a guideline for further exploration.

Another potential source for the observed discrepancy in Figure 5 may be the grid resolution. However, as seen from Table 1, the grid resolution near the bottom adjacent to the pile where the horseshoe vortex forms appears to be sufficiently small in the $\mathrm{x}$-direction, considering the $\mathrm{x}$ extent of the horseshoe vortex being typically $0.25 D$ for $K C=10$ for waves and $0.5 D$ for currents (from Sumer et al.'s experiments, 1997). The grid resolution in the z-direction (see Table 1) is also sufficiently fine, again, considering the z-extent of the horseshoe vortex being typically $0.1 D$ for $K C=10$ (from Sumer et al.'s experiments, 1997) and 0.3D for currents (from Roulund et al.'s experiments, 2005). Therefore, the present grid resolution appears to be adequate to resolve the horseshoe vortex in all cases (current and waves). The authors have, in a previous study (Baykal et al. 2015), tested finer meshes especially for hydrodynamic comparisons. Since not a significant improvement (less than 5\%) has been achieved in terms of the bed shear stresses for that particular finer mesh, a direct comparison of bed shear stresses between meshes has not been presented in the present study. We also note that such finer meshes with the number of cells of $\mathrm{O}\left(10^{6}\right)$ or larger were not used, as such fine meshes could not be utilized in the morphology 
simulations. We further note that similar discrepancies in bed stresses have also been observed in Roulund et al.'s study (2005), in which a mesh with approximately $8 \times 10^{5}$ cells for the rigid bed calculations was used.

The distance $x_{s}$ in Figure 1 is the length characterizing the size of the horseshoe vortex. This length can be determined from the zero-crossings of the bed shear stress variations in Figure 5. The figure indicates that the present numerical model is able to predict the size of the horseshoe vortex reasonably well, as the zero crossings from the numerical model appear to be rather close to (or coincide with) those from the laboratory experiments. Furthermore, the numerical results in Figure 5 also show that $x_{s}$ increases with increasing values of the $K C$ number, in agreement with the existing information in the literature (see, e.g.. Sumer and Fredsøe, 2002, Fig. 3.14).

\subsection{Lee-wake vortices}

The lee-wake vortices are formed mainly due to the rotation in the boundary layer over the surface of the pile. The shear layers emanating from the side edges of the pile roll up to form these vortices in the lee-wake of the pile. The emanating position of these shear layers on the cylinder, the boundary layer separation, changes over the height of the cylinder when the approach-velocity profile is not uniform over the depth of the domain (see Baykal et al., 2015, Fig. 4). From dimensional grounds, the non-dimensional quantities describing the lee-wake flow around a smooth pile depend on: $\operatorname{Re}_{D}$ in the case of steady currents, and $\mathrm{Re}_{D}, \mathrm{KC}$ in the case of waves.

For steady currents, the flow in the lee-wake becomes unsteady, and the vortex shedding begins to occur with vortices shed alternately at either side of the pile when the Reynolds number $\operatorname{Re}_{D}$ reaches the value 40 . In the present study, the Reynolds number is $\operatorname{Re}_{D}=1.7 \times 10^{4}$, and this Reynolds number implies that the vortex shedding occurs in the turbulent regime (see, e.g., Sumer and Fredsøe, 2006). The present case (the pile mounted on a flat, rigid bed, and subjected to a steady current, Test 1 , Table 2) has been investigated in greater detail in our previous study, Baykal et al. (2015). Figure 3 illustrates the way in which the vortex shedding takes place. Baykal et al.'s (2015) numerical results for this particular case have shown the following: (1) The boundary layer over the pile surface separates much earlier close to the bed than at higher 
elevations (Baykal et al., 2015, Fig. 4), mainly due to the smaller velocities in the approach flow close to the bed. (2) Because the velocities close to the bed are smaller, the magnitude of the vorticity supply into the lee-wake vortices close to the bed becomes less than the upper parts of the domain. (3) An important implication of the latter is that the vortex shedding "slows down" close to the bed, and thus, the shed vortices across the height of the pile are broken apart into two cells in the vertical direction, one near the bed, the rest across the entire height of the pile. The "two-cell" structures of the shed vortices can be seen more visibly in Baykal et al. (2015, Fig. 7).

Figure 4 illustrates the way in which the vortex shedding occurs in the case of oscillatory flow, $K C=20$ (Test 14, Table 2). Figure 4 shows that the two-cell shedding regime, observed for the steady-current case, practically ceases to exist in the case of waves (although there is a slight tendency that the vortices seem to have small tails, close to the bed, towards the end of the half period, Figure $4 \mathrm{c}$ and d) (c.f., Figs. 3 and 4). The fact that the two-cell shedding regime practically disappears in waves is linked to the following two effects: (1) the small boundary layer thickness experienced in the wave boundary layer; and (2) the almost uniform separation across the height of the pile, in contrast to the steady current situation.

The lee-wake vortices for the steady current test, and the two wave tests $(K C=10$ and $K C=20)$ are visualized in an accompanying video (see electronic supplementary material-1 of the paper). The video is actually composed of three videos, corresponding to the steady current test, and the two wave tests with $K C=10$ and $K C=20$. The videos visualize the time evolution of the iso-surfaces of the vorticity in the $z$-direction $\left(\omega_{z}\right.$ in $\left.1 / \mathrm{s}\right)$ around the pile. The flow visualization in the videos clearly reveal the nonexistence of the two-cell shedding regimes in the case of the wave tests (c.f., the steady current and the wave videos). It may be noted that the videos also show that one vortex is shed in each half-period for $K C=10$, whereas two (or occasionally three, albeit not very frequently) vortices are shed for each half period for $K C=20$, in-line with Williamson (1985) and An et al. (2015). This is an interesting result in the sense that the lee-wake vortex-flow regimes around a wall-mounted vertical cylinder in waves are very similar to those for a free cylinder. 


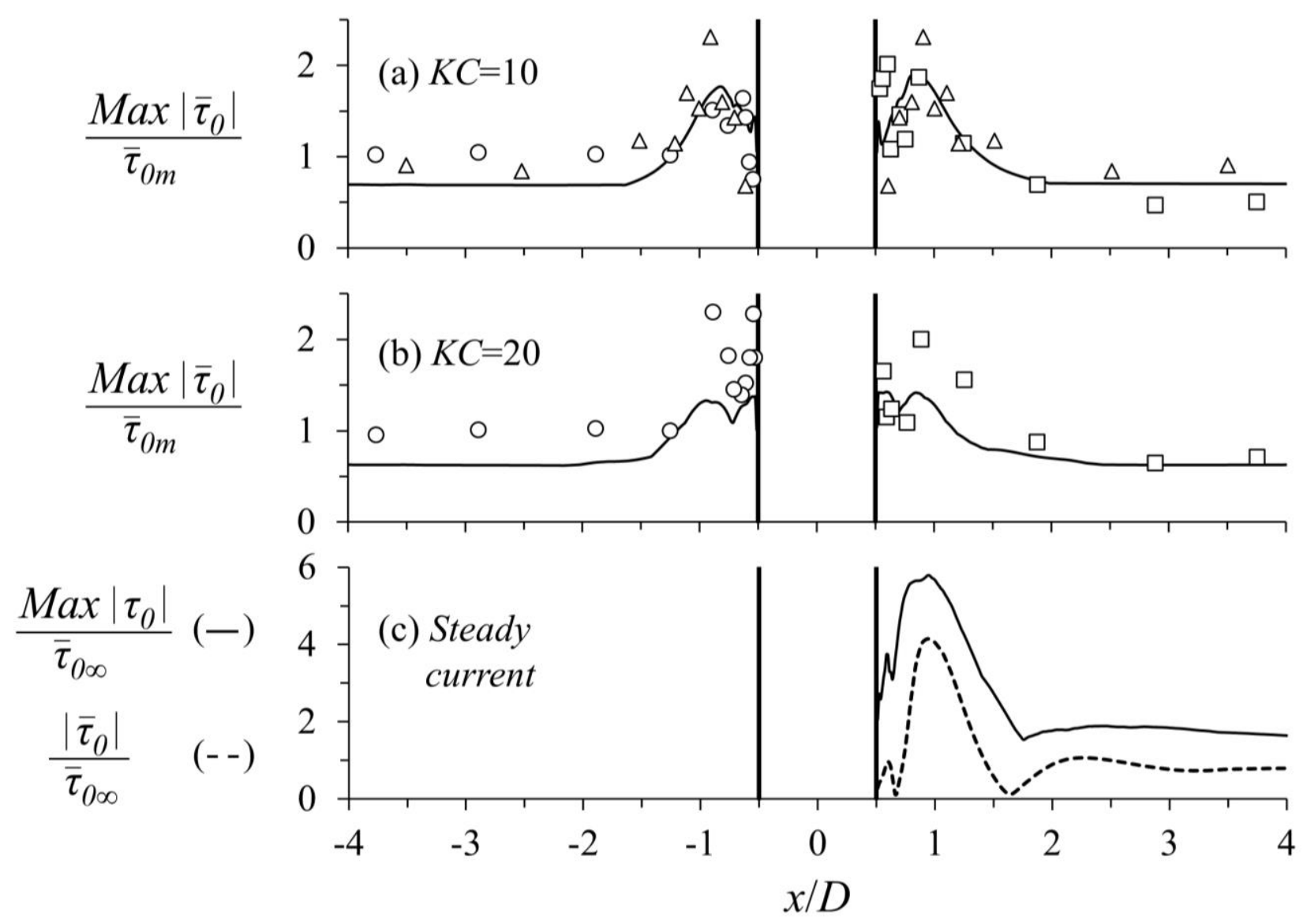

Figure 6. Amplification of the maximum bed shear stress along the $\mathrm{x}$-axis at the lee-side of a circular pile. Circles, in front of the pile; squares, behind the pile, the wave-flume experiments; triangles, the oscillatory-tunnel experiments, all from Sumer et al. (1997). Solid line, the present numerical model in the front of the pile (at the phase values $\omega t=270^{\circ}$ for $x / D<0$ ) and at the back of the pile (at the phase values $\omega t=90^{\circ}$ for $x / D>0$ ) for (a) $K C=10$, Test 8 in Table 2 and (b) $K C=20$, Test 14 in Table 2. Solid line in (c) shows the maximum instantaneous bed shear amplification, dashed line the phase-averaged bed shear stress amplification for steady current.

Figure 6 depicts the amplification of bed shear stress distribution along the $x$-axis in the lee-wake area for (a) $K C=10$ and (b) $K C=20$, and (c) for steady current. Here, the present model results (solid lines for the front side of the pile and dashed lines for the back side of the pile in (a) and (b)) are compared with the experimental data of Sumer et al. (1997) (see Fig.17c and d on p. 58 and the Test 8 and 14 data on p. 66 and Test 64 data in p. 69 in Sumer, 1997) for $K C=10$ and $K C=20$. Figure $6(\mathrm{c})$ depicts the maximum value of the amplification at the lee side, $\operatorname{Max}\left|\tau_{0}\right| / \bar{\tau}_{0 \infty}$, and the mean value of bed shear stress amplification at the lee side of the pile, $\left|\bar{\tau}_{0}\right| / \bar{\tau}_{0 \infty}$. As seen 
from Figure 6, the bed shear stresses computed by the present numerical model are in fairly good agreement with the measurements, especially away from the cylinder $|x / D|>\mathrm{O}(1)$. Also, the results of the numerical simulations show similar behavior with the experiments as the extent of the area influenced by the lee-wake increases with increasing $K C$. The amplification in the bed shear stress with respect to its undisturbed value can be as high as 2 for $K C=10$ and 1.5 for $K C=20$ but it gets even higher, $\operatorname{Max}\left|\tau_{0}\right| / \bar{\tau}_{0 \infty} \approx 6$, for the steady current case. It may be noted that such an increase in bed shear stress for steady current is also reported by Sumer et al. (1997).

Finally, as mentioned earlier (Section 1), in the lee wake, further downstream the pile, a pair of counter-rotating streamwise vortices also comes into existence (Figure 1). This feature of the flow has first been observed in the laboratory by Petersen (2014) (also reported in Petersen et al., 2015) in the context of the so-called edge scour at the downstream side of a scour protection structure around a circular pile. The aforementioned counter-rotating vortices have been visualized numerically by Baykal et al. (2015, see Figs. 11 and 12). These vortices have been discussed in Baykal et al. (2015) in greater detail, and therefore it will not be pursued here any further.

In the case of waves, the streamwise counter-rotating vortices are expected not to be very significant, as the approach-flow boundary layer thickness is rather small. In fact, the present wave simulations do indicate that these vortices are almost nonexistent.

\subsection{Amplification of bed shear stress}

The distribution of the bed shear stress along the streamwise axis has been discussed earlier in conjunction with the horseshoe vortex (Figure 5) in Section 4.1, and with the lee-wake (Figure 6) in Section 4.2.

Figure 7 gives the full picture of the bed shear stress in the form of contour plots for the studied cases (the steady current, Test 1, Table 2; and for the wave cases, $K C=10$, Test 8 , and $K C=20$, Test 14 in Table 2). The amplification of the bed shear stress in the figure is defined as the ratio of maximum magnitude of the bed shear stress vector to the value of to the maximum value of the undisturbed bed shear stress in the case of waves $\left(\operatorname{Max}\left|\bar{\tau}_{0}\right| / \bar{\tau}_{0 m}\right)$ and the undisturbed bed 
shear stress in the case of steady current $\left(\operatorname{Max}\left|\bar{\tau}_{0}\right| \bar{\tau}_{0 \infty}\right)$ obtained from the present model and that from experiments of Sumer et al. (1997) (see Fig. 22c \& d on p. 62) for $K C=10$ and $K C=20$, and Hjorth (1975) for steady current (see Fig.6.18b on p. 133 of Hjorth, 1975).
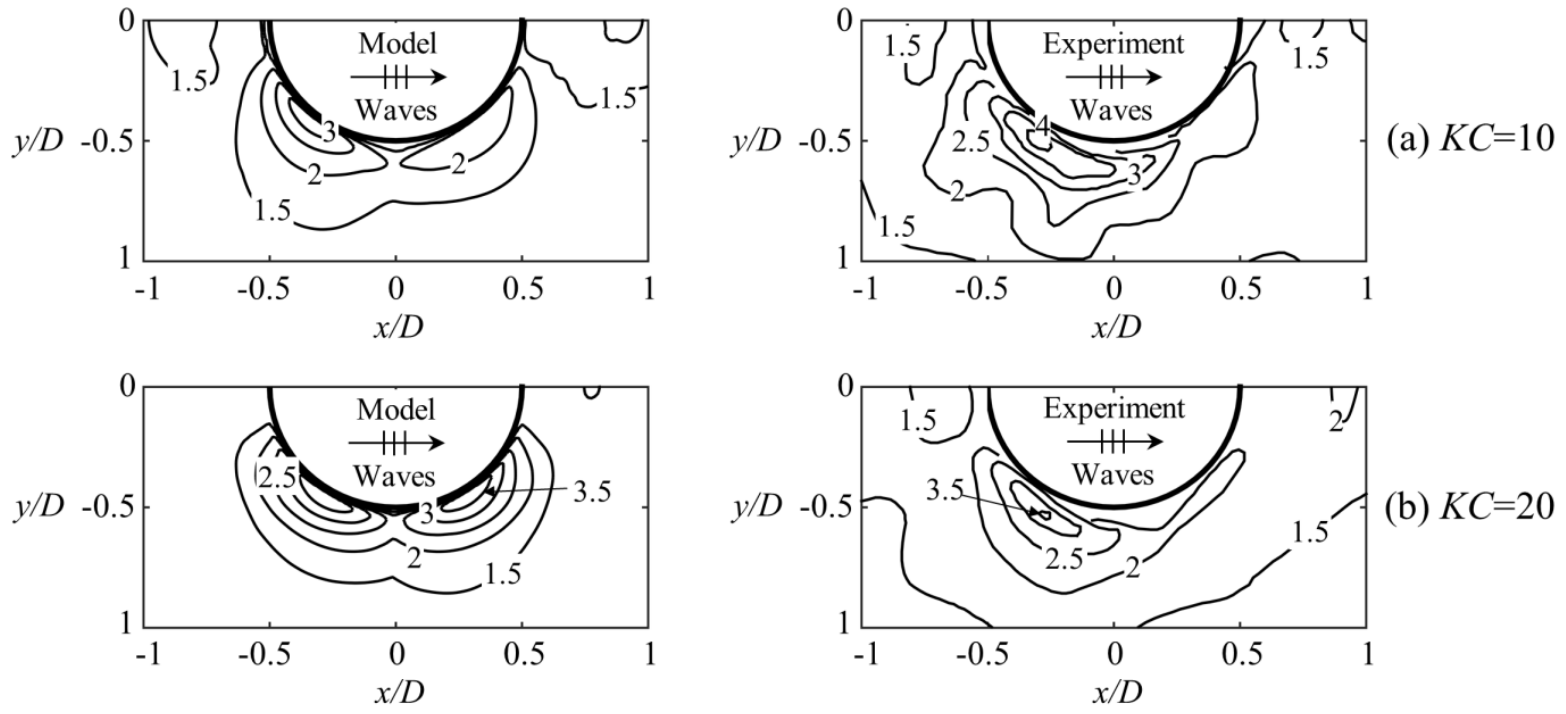

(b) $K C=20$
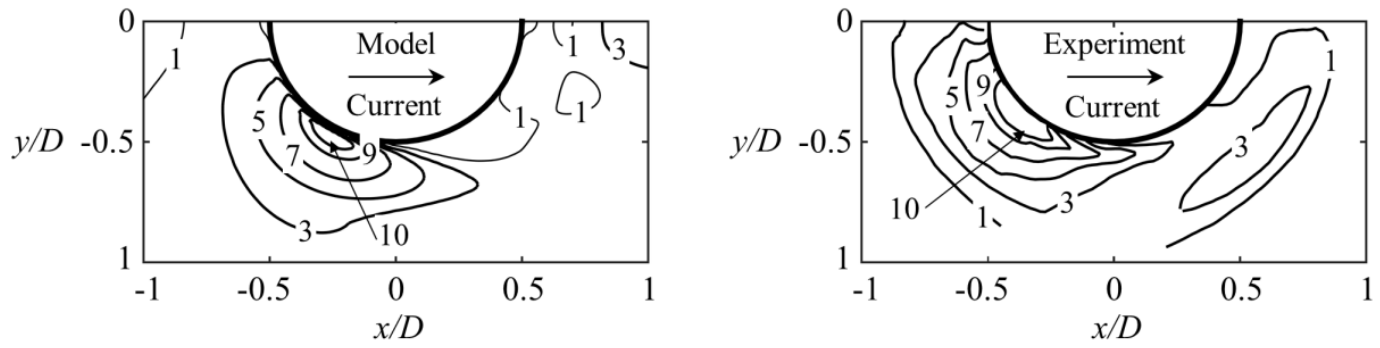

(c) Steady current

Figure 7. Amplification of the bed shear stress around the pile for (a) the oscillatory flow $K C=10$ (Test 8, Table 2), (b) $K C=20$ (Test 14, Table 2), and (c) steady current (Test 1, Table 2). Experiments for (a) and (b) from Sumer et al. (1997), and for (c) from Hjorth (1975). The steady current case adapted from Baykal et al. (2015). Model results are given on the left, and the experimental results are given on the right.

First of all, the asymmetry observed in the experimental data in Figure 10a and $\mathrm{b}$ is due to the asymmetry in the waves between the crest and trough half periods. We note that comparison between the simulations and the experiments would be on the same basis only if we implemented the same asymmetric oscillatory flow in the simulations. However, this idea has not been pursued further, as the focus of the present study was the morphology. 
Secondly, the numerical results and the experiments seem to be in agreement in terms of the magnitude of the amplification and also in terms of the location of the maximum value of the amplification.

Thirdly, the numerical results reveal the experimental finding that while the maximum amplification in the bed shear stress around the pile remains at $\mathrm{O}(3-4)$ in the wave cases, it can be as much as $\mathrm{O}(10)$ in the case of the steady current. In the former case, the maximum amplification is essentially governed by the contraction of the streamlines near the sides of the pile. In the steady current case, however, it is governed by partly contraction of streamlines, and partly by the strong presence of the horseshoe vortex, as pointed out by Sumer et al. (1997).

As a final note, the capability of the present numerical model in predicting the bed shear stresses around the pile may prove useful when considering its application in the design of scour protection around piles.

\section{Scour simulations}

Three kinds of scour tests are carried out in the numerical simulations: a steady current test, and two wave tests (Table 3). The test conditions depicted in Table 3 are identical to those of Sumer et al.'s (2013a) laboratory experiments carried out in a current/wave flume, with test numbers Test 1, Test 6 and Test 9 in Sumer et al. (2013a). The pile diameter is $D=40 \mathrm{~mm}$, and the grain size is $d=0.17 \mathrm{~mm}$ (corresponding to fine sand) (Sumer et al., 2013a).

In Table 3, Test 6 and Test 9, the wave experiments of Sumer et al. (2013a), were carried out in irregular waves. The quantity $U_{m}$ in Table 3 in Sumer et al.'s (2013a) experiments is the orbital velocity at the bed level (corresponding to the maximum value of the orbital velocity) calculated from the spectrum function of $U$

$$
U_{m}=\sqrt{2} \sigma_{U}
$$

with $\sigma_{U}$ defined as 


$$
\sigma_{U}=\int_{0}^{\infty} S_{U}(f) d f .
$$

Here $S_{U}(f)$ is the spectrum of the measured orbital velocity at the bed $U$ and $f$ the frequency. Note that $U_{m}=\sqrt{2} \sigma_{U}$ becomes identical to the maximum value of the bed orbital velocity in the case of small-amplitude sinusoidal waves. The quantity $U_{m}$ is the maximum value of the free stream velocity of the regular waves in the numerical tests, defined by Eq. 26 . The quantity $T_{w}$ in Table 3 is the wave period corresponding to the peak frequency of Sumer et al.'s (2013a) experiments, $T_{w}=1 / f_{p}$ (namely, the peak period), and the quantity $U_{f m}$ is the maximum value of the friction velocity, calculated from

$$
U_{f m}=\sqrt{\frac{f_{w}}{2}} U_{m},
$$

in which $f_{w}$ is the wave friction factor. Sumer et al. (2013a) gives the quantity $U_{f m}$ in Table 3 and Table 4, computing the friction factor from $f_{w}=2 /(\operatorname{Re} \delta)^{0.5}$, (Fredsøe and Deigaard 1992), based on the fact that the bed in the experiments is hydraulically smooth $\left(d_{50} U_{f m} / v<5\right)$ and the wave boundary layer is in laminar regime $\left(\operatorname{Re}_{\delta}<1.5 \times 10^{5}\right)$.

Table 3. The test conditions for scour simulations. All cases use pile diameter $D=40 \mathrm{~mm}$ and sand grain size $d=0.17 \mathrm{~mm}$.

\begin{tabular}{ccccccc}
\hline $\begin{array}{c}\text { Test No. of } \\
\begin{array}{c}\text { Sumer et al.'s (2013a) } \\
\text { current/wave flume } \\
\text { expts. }\end{array}\end{array}$ & $\begin{array}{c}\text { Keulegan- } \\
\text { Carpenter } \\
\text { Number, } \\
K C\end{array}$ & $\begin{array}{c}\text { Mean flow } \\
\text { velocity, } \\
V(m / s)\end{array}$ & $\begin{array}{c}U_{m} \\
(\mathrm{~m} / \mathrm{s})^{(1)}\end{array}$ & $\begin{array}{c}\text { Wave } \\
\text { period } \\
T_{w}(\mathrm{~s})\end{array}$ & $\begin{array}{c}U_{f} \\
\left(\text { or } U_{f m}\right) \\
(\mathrm{m} / \mathrm{s})\end{array}$ & $\begin{array}{c}\text { Shields } \\
\text { parameter, } \\
\theta\end{array}$ \\
\hline 1 & $\infty$ (Current) & 0.413 & - & - & 0.019 & 0.13 \\
\hline 6 & 20 & - & 0.20 & 4.0 & 0.016 & 0.09 \\
\hline 9 & 10 & - & 0.225 & 1.79 & 0.020 & 0.15 \\
\hline
\end{tabular}

(1): $U_{m}$ is the maximum value of the undisturbed orbital velocity at the bed in Sumer et al.'s (2013a) wave-flume experiments (see the text for details), or the maximum value of the free stream velocity of the oscillatory flow in the numerical simulations, defined by Eq. 26.

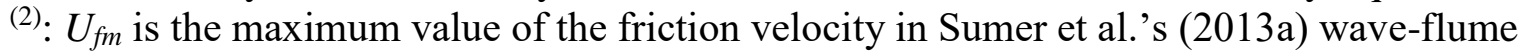
experiments, or the maximum value of the friction velocity of the oscillatory flow in the numerical simulations. 
The scour in all three tests in Table 3 occurred in the live-bed regime in Sumer et al.'s (2013a) experiments, as revealed by Sumer et al.'s visual observations. This is also revealed by the fact that the Shields parameter (Table 3) is larger than the critical value for the initiation of motion on the bed, $\theta>\theta_{c r}(=0.05$, corresponding to the grain Reynolds number of about 4 , see, e.g., Sumer and Fredsøe, 2006, Fig. 1.2).

Scour around a circular pile in steady currents has been studied numerically by several researchers, Roulund et al. (2005) and Baykal et al. (2015), among others (an extensive list of these studies has been given in Section 1). In the present investigation, Roulund et al.'s (2005) study has been extended to waves. One test in the investigation, however, is devoted to a steady current simulation (Test 1, Table 3), in order to utilize the numerically obtained scour hole in the steady current case for backfilling simulations in waves in the tests Test 27 and Test 32 (Table 4). We shall not present the results associated with this steady-current test since the numerical simulation of the scour process, including various aspects of the scour process such as the time evolution, the scour depth, the time scale, etc., has been covered extensively in our earlier studies, Roulund et al. (2005) and Baykal et al. (2015). Therefore, the following paragraphs will focus on the results of the wave-induced scour simulations. Figures 8 and 9 show sequences of pictures illustrating the time evolution (from an initially flat bed) of the scour hole from the wave simulations, Figure 8 for $K C=10$ (Test 9, Table 3), and Figure 9 for $K C=20$ (Test 6, Table 3). 


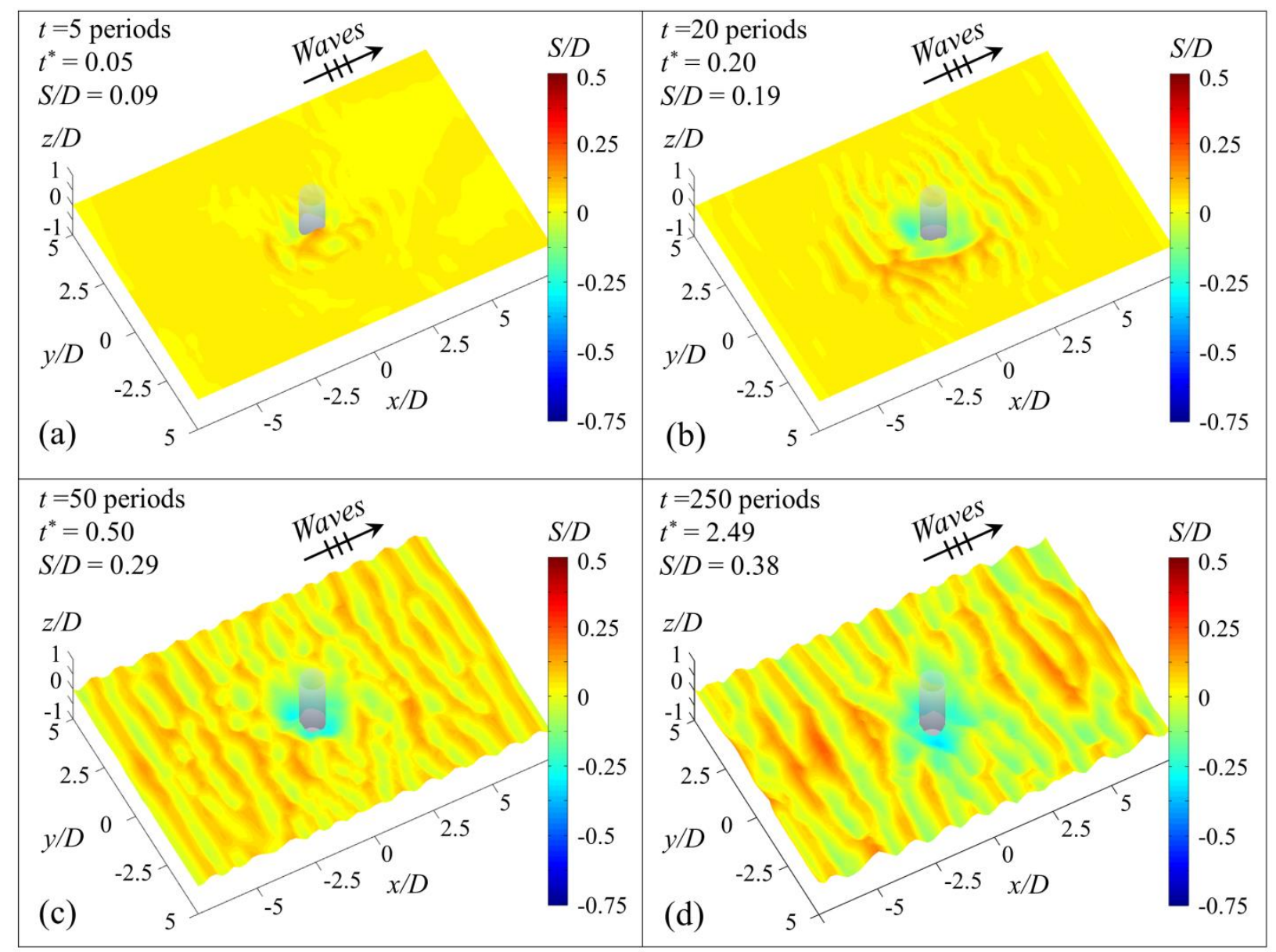

Figure 8. Scour development for $K C=10$, Test 9 in Table 3. Model results. Scour depth in front of the cylinder, normalized by the pile diameter, is indicated in the panels. 


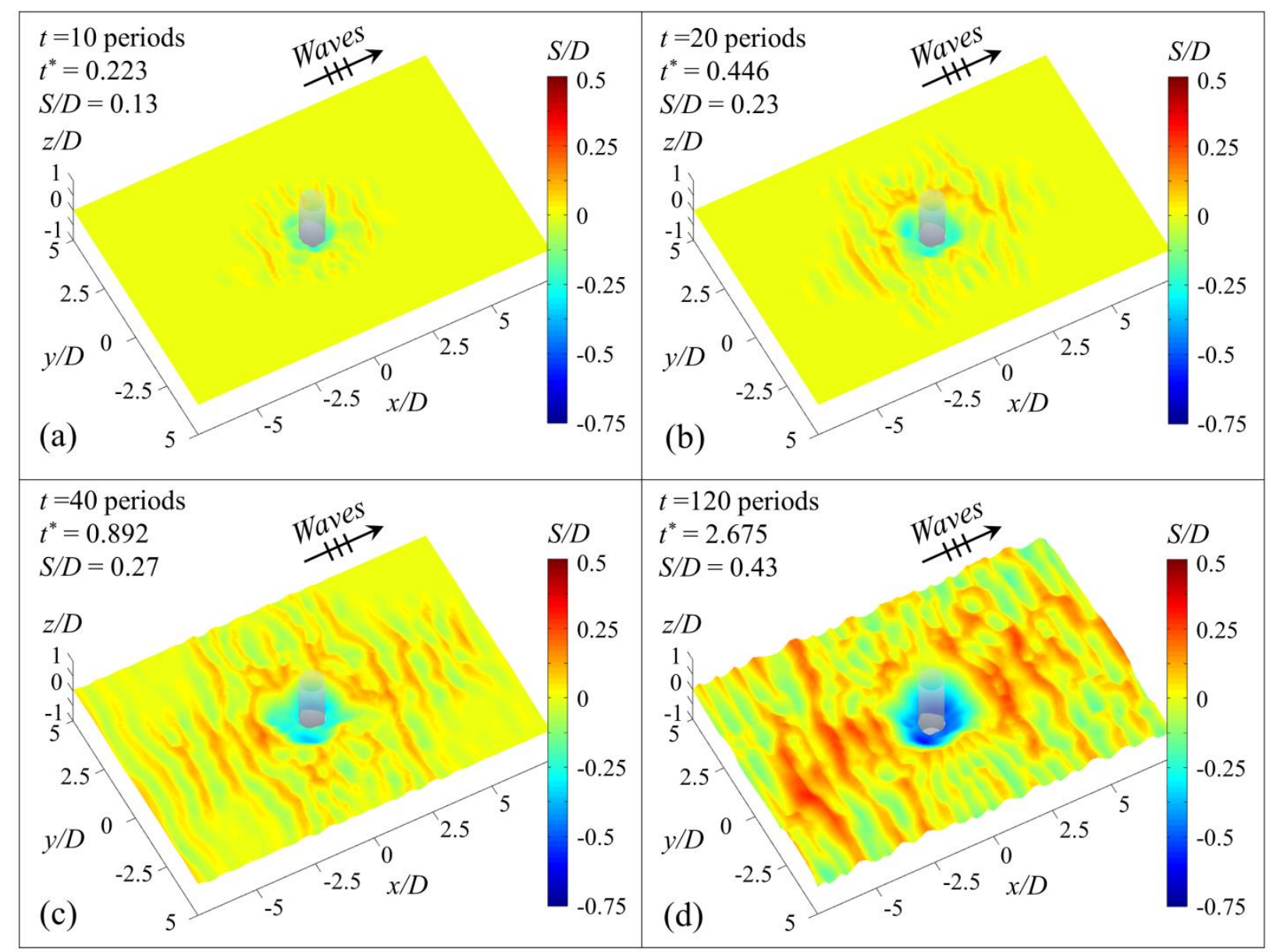

Figure 9. Scour development for $K C=20$, Test 6 in Table 3. Model results. Scour depth in front of the cylinder, normalized by the pile diameter, is indicated in the panels.

Figure 10, on the other hand, shows the time development of the scour depth (both at the offshore side and at the onshore side of the pile) in these tests. In Figure 10, the equilibrium scour depths obtained in Sumer et al.'s (2013a) wave experiments are also plotted as reference lines (dashed lines in the figure). Furthermore, Figure 11 depicts the scour-hole profiles along the offshoreonshore symmetry axis, at later stages of the scour process, namely at $250 T_{w}\left(t^{*}=2.5\right)$ for $K C=10$, and at $160 T_{w}\left(t^{*}=3.5\right)$ for $K C=20$. Here $t^{*}$ is the non-dimensional time defined by

$$
t^{*}=\frac{\sqrt{g(s-1) d^{3}}}{D^{2}} t \text {. }
$$

Now, from Figures 8-11, the following observations can be made: 
It is seen from Figures 8-10 that the scour evolves towards the equilibrium stage through a transitional period. Figure 10 further indicates that the asymptotic values attained by the numerical scour-depth time series (the equilibrium scour depths) are not radically different from those of Sumer et al.'s experiments (2013a) (horizontal dashed lines).

Secondly, ripples, resolved in the present simulations, begin to form on the bed surface from the early stages of the simulation, and eventually reach an equilibrium stage as the test continues. In the initial stage (Figures 8a and b, and Figures 9a and b), the ripples are formed as a result of the instability mechanism associated with the sediment bed in an otherwise undisturbed flow situation (without the pile). In the later stages, the ripple growth is heavily influenced by the vortex flow around the pile. When closely inspected, the ripple length $(\lambda)$ in the initial stage appears to be smaller in Figures $8 \mathrm{a}$ and b $(K C=10$ case) than in Figures $9 \mathrm{a}$ and b $(K C=20$ case), namely $\lambda / D=0.8$ and 1.1 for $K C=10$ and 20 , respectively. These values are comparable with the values obtained from the empirical relation for vortex ripples $\lambda \approx 1.2 a$ (e.g., Brøker, 1985), or $\lambda / D=[1.2 /(2 \pi)] \cdot K C$, namely $\lambda / D=2$ and 4 for $K C=10$ and 20 , respectively. Here, $a=U_{m} T_{w} /(2 \pi)$ is the amplitude of the orbital motion of particles in the free stream. The ripple lengths in the simulations appear to be a factor of 2-4 smaller than the values from Brøker's (1985) relation. This is because the simulation values are associated with the initial stages of the ripple growth, and, in an instability mechanism, the characteristics of bed forms in the initial stages are always significantly smaller than those of the final stages (see, e.g., Sumer and Bakioglu, 1984, p. 189). As already mentioned, in the present problem (ripple formation in the disturbed case), the ripple growth is, in the final stages, heavily influenced by the vortex flow around the pile, and therefore no direct comparison is made between the equilibrium ripple lengths exhibited in Figures $8 \mathrm{~d}$ and $9 \mathrm{~d}$, and Brøker's relation $\lambda \approx 1.2 a$.

Thirdly, unlike the scour process exhibited in Figure $9(K C=20)$, there is an apparent asymmetry in the early stages of the scour process in the case of $K C=10$ (see the panel corresponding to $t=5$ periods ( or $t^{*}=0.05$ ), Figure 8a). This is linked to the presence of the transverse vortex street for the vortex shedding regime $7<K C<13$ (Williamson, 1985; Sumer and Fredsøe, 2006). However, this asymmetry disappears (Figure 8b-d) as the scour process continues, with the bed profile changing from flat to the usual inverse truncated-cone shape. The flow visualization of 
this test does show the presence of the transverse vortex shedding in the early stages of the scour process (for the first 30 periods of the test). This exercise reveals that the shedding changes sides for larger times.

Fourthly, the computed equilibrium scour depths (Figure 10) show a "dynamic" behavior. As noted by Fuhrman et al. (2014) in conjunction with their study on scour and backfilling beneath a marine pipeline, this is likely due, partly, to the use of instantaneous morphological updating, evolution of small scale bed features inside the scour hole, and resulting changes in the hydrodynamic flow conditions (i.e., vortex shedding regimes) updated simultaneously around the pile in time.

Finally, Figure 11 indicates that, unlike the steady-current scour case (see Fig.15 in Baykal et al. 2015, and also see the initial scour hole in Fig. 19, used in the backfilling simulations), the scour holes at both sides of the pile (offshore and onshore sides) are symmetric, and the scour depths and the slopes of the scour hole at both sides of the pile are similar, in agreement with experimental observations.
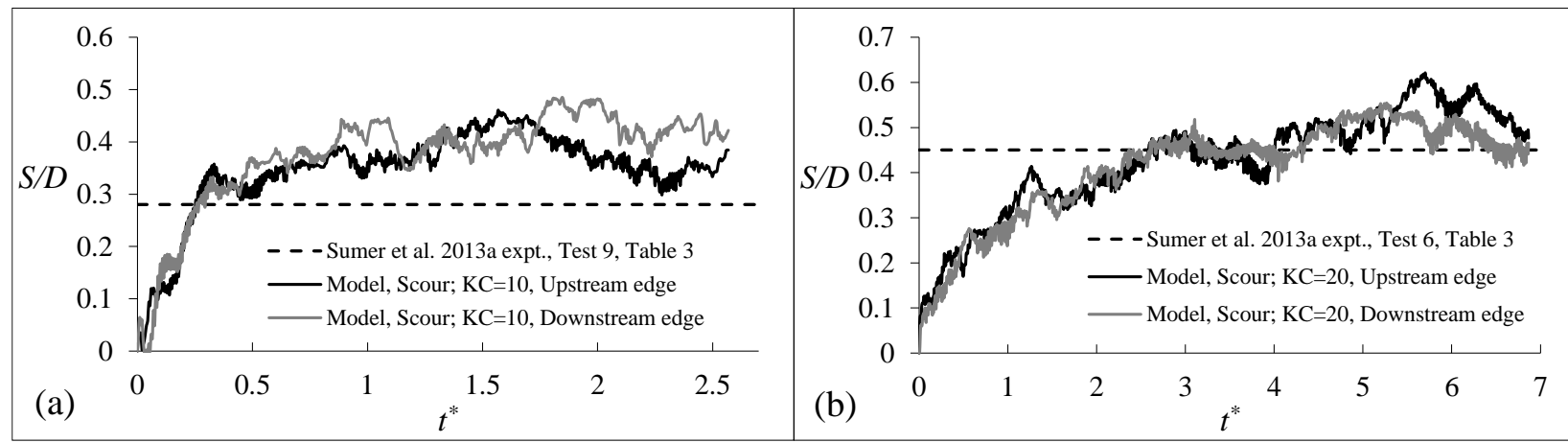

Figure 10. Scour development for (a) $K C=10$, Test 9, and (b) $K C=20$, Test 6 in Table 3. Solid black line: scour depth at the upstream edge of the pile. Solid gray line: scour depth at the downstream edge of the pile. Dashed lines are equilibrium scour depths reached in Sumer et al.'s (2013a) experiments. 


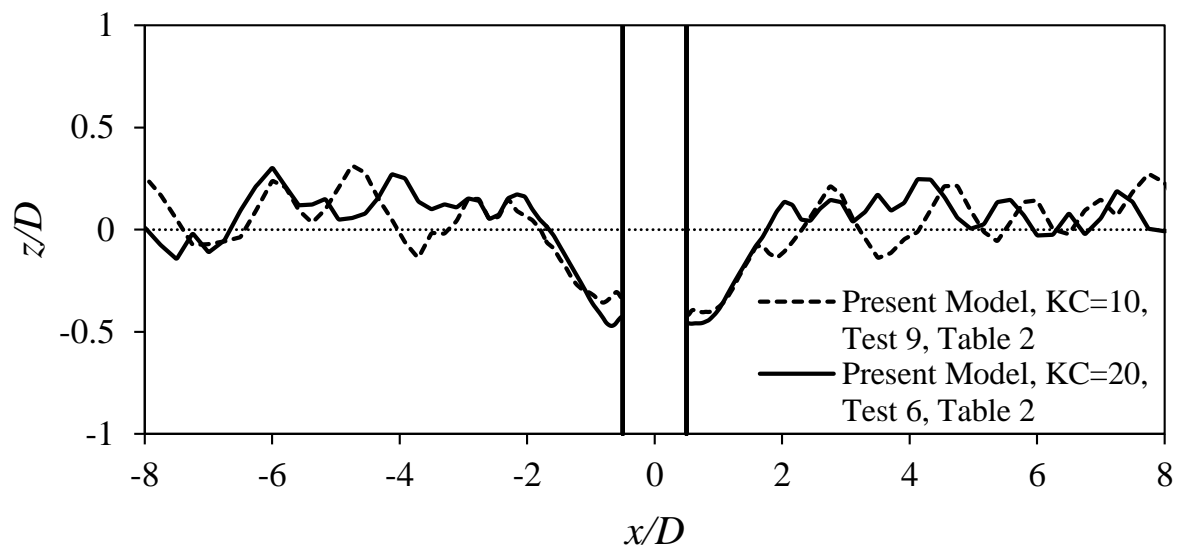

Figure 11. Equilibrium scour profile along the length of the longitudinal symmetry axis for $K C=10$ (dashed line, Test 9 in Table 3 ) wave scour after $250 T_{w}\left(t^{*}=2.5\right.$ ) and for $K C=20$ (solid line, Test 6 in Table 3) wave scour after $160 T_{w}\left(t^{*}=3.5\right)$ of simulation time.

Figure 12 displays a sequence of pictures where (1) the flow is visualized by the vorticity in the vertical direction, $\omega_{z}$, and (2) the sediment is visualized by the iso-surfaces of concentration corresponding to a sediment concentration value of $c=0.002$ by volume (in solid yellow). The oscillatory flow is run under the same test conditions as in Test 14 in Table 2, $K C$ being $K C=20$. The threshold values used to plot the iso-surfaces of vertical component of the vorticity, the sediment concentration, and the upward-directed pressure gradient depicted in the upcoming Figure 13 and the second accompanying video, are determined by trial and error, so that one gets the clearest visualization of the movement of the sediment volume synchronized with that of the lee-wake vortices. In Figure 12, using a right hand rule, the positive values of vorticity depicted in transparent green color states a counter-clockwise rotation of the vortex, and vice-versa for the blue transparent, negative values. The figure clearly shows the way in which the scour process occurs; namely, the vortex forming (and eventually shedding) in the lee wake (Vortex A, Figure 12a) picks up the sediment, and sweeps it into its core; it then carries the sediment away from the pile while it is convected downstream (Figure 12b and c); and eventually the sediment is deposited further downstream, coinciding with the time when Vortex A ceases to exist (Figure $12 \mathrm{~d}$ ), thus causing a net scour around the pile. This observation is in complete agreement with the mechanism explained by Sumer et al. (1993) on the basis of their laboratory observations of wave scour (included also in Sumer and Fredsøe, 2002, pp. 188-189). 


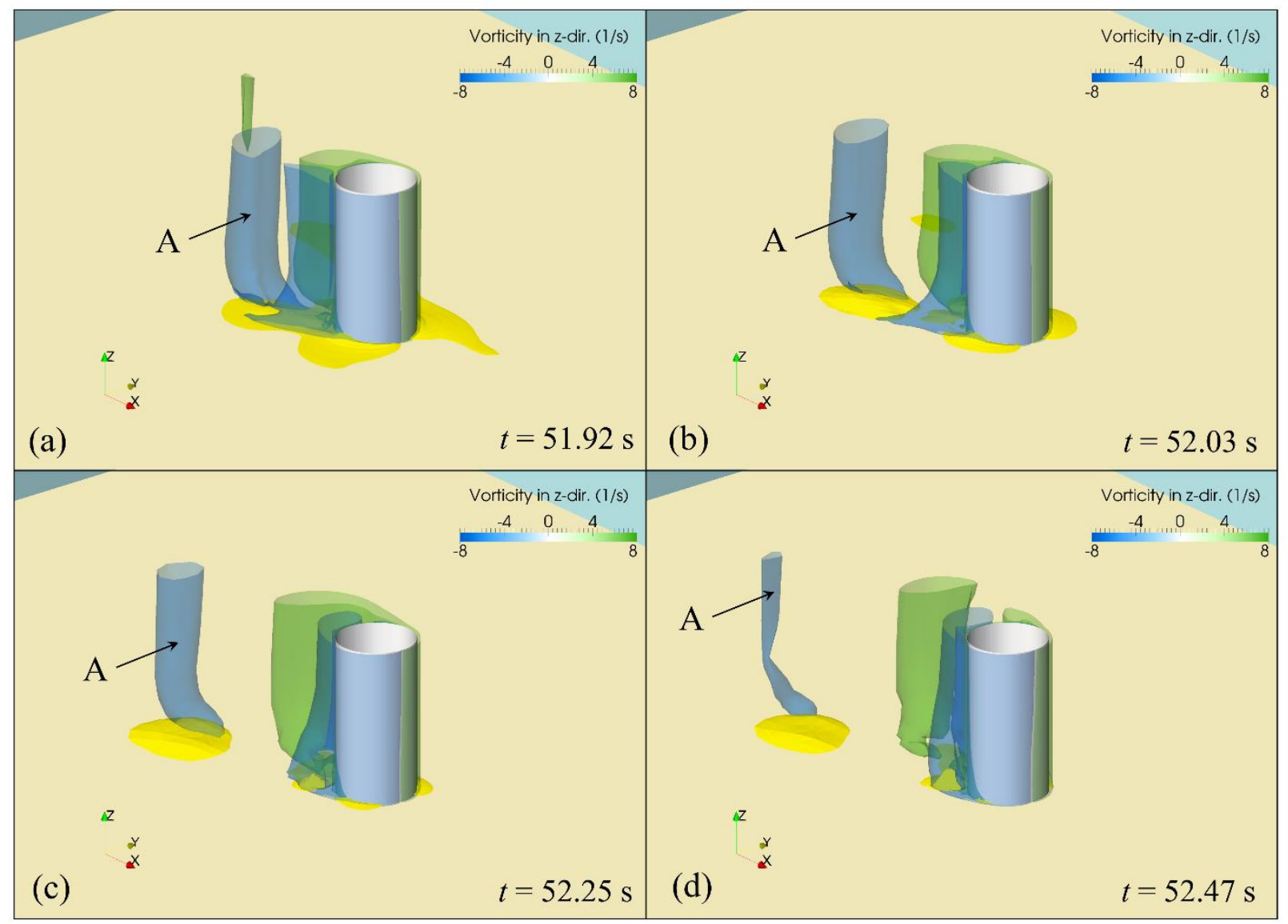

Figure 12. Numerical flow visualization for the lee-wake vortices as in transparent blue and green iso-surfaces of vertical component of vorticity $\left(\omega_{z}\right.$ in $\left.1 / \mathrm{s}\right)$, and the sediment volume depicted in the form of iso-surfaces of sediment concentration (solid yellow iso-surfaces with concentration by volume, $c=0.002$ ) around the cylinder at different phases of oscillatory flow, run at the same test conditions as in Test 14 in Table 2, $K C$ being $K C=20$. The times shown in the figure are given in seconds, and correspond to times after the start of simulation, $t=0 \mathrm{~s}$.

Figure 13 shows the results of two additional flow visualizations: (1) Figure 13a displays the contour plot of the Shields parameter. (2) Figure 13b displays the iso-surface of the upwarddirected pressure gradient $\left(-\frac{\partial(p / \gamma)}{\partial z}\right)=0.1$, along with the shed vortices visualized by the plot of the vertical component of the vorticity in both Figure 13a-b. Note that the "snapshots" in Figure 13 correspond precisely to the same time instant in Figure 12b. Here, too, the threshold values used to plot the iso-surfaces of vertical component of the vorticity, the sediment concentration, and the upward-directed pressure gradient depicted in Figures 12 and 13 and the second 
accompanying video, are determined by trial and error, so that one gets the clearest visualization of the movement of the sediment volume synchronized with that of the lee-wake vortices.

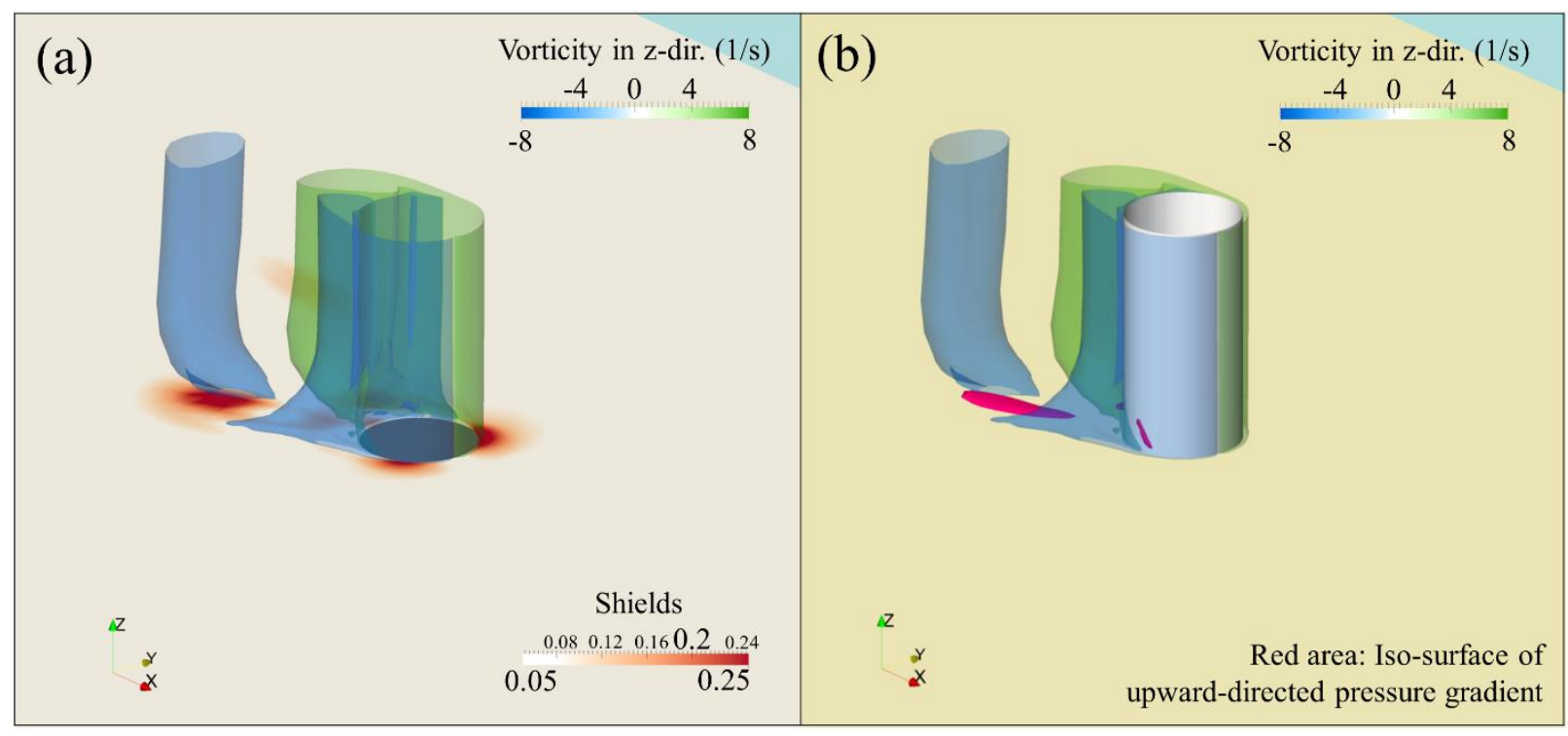

Figure 13. Numerical flow visualizations for the same test as in the previous figure, corresponding to precisely the same time instant in Figure $12 \mathrm{~b}$, namely at $t=52.03 \mathrm{~s}$. (a) The contour plot of the Shields parameter on the bed surface. (b) The iso-surface of the vertical pressure gradient over the bed, corresponding to $\left(-\frac{\partial(p / \gamma)}{\partial z}\right)=0.1$ (the red color). The shed vortices are visualized by the plot of the vertical component of the vorticity $\left(\omega_{z}\right.$ in $\left.1 / \mathrm{s}\right)$.

The Shields parameter plotted in Figure 13a is computed using the magnitude of the bed shear stress vector, see Eq. 20. Here, the minimum value of Shields parameter plotted is defined to be equal to the critical value of Shields parameter, taken as $\theta_{c r}=0.05$ in the simulations. Regarding the vertical pressure gradient $\left(-\frac{\partial(p / \gamma)}{\partial z}\right)$ in Figure 13, this quantity essentially represents a lift force, the lift force being directed upwards if $\left(-\frac{\partial(p / \gamma)}{\partial z}\right)>0$, and downwards if $\left(-\frac{\partial(p / \gamma)}{\partial z}\right)<0$. It may be noted that this quantity, namely the vertical pressure gradient, studied earlier in the past by researchers such as Engelund (1970) and Sumer (1984) in the context of lift forces on nearbed sediment particles, and by Jensen et al. (2014) in the context of oscillatory turbulent wave boundary layers. It is important to note that all the content here in the present study are vertical pressure gradient in the water column, and hence it is different from that studied previously in the 
context of pore pressure gradient reported in publications such as Sumer et al. (2006), Sumer et al. (2011 and 2013b) and Sumer (2014).

Now, Figure 13 clearly shows that the picking-up of the sediment and its sweeping into the shed vortex (and presumably its transport in the downstream direction) (Figure 12a-d) is closely associated with the large values of the bed shear stress and the upward-directed pressure gradient $\left(-\frac{\partial(p / \gamma)}{\partial z}>0\right)$ created underneath the shed vortex (Figure 13a-b). The figure implies that, with the formation of Vortex A (Figure 12a), the bed sediment will be swept into Vortex A due to the very high Shields-parameter values (very high shear stresses) produced by this vortex. At the same time, the bed sediment will undergo a large upward-directed pressure gradient force induced by the same vortex, Vortex A. As a result of the combined action of these two effects, the sediment will be "sucked" into the main body of the vortex, and carried away within the body of the vortex, as the vortex is convected downstream, as revealed clearly by Figure 12.

It is important to note that the aforementioned process is implicitly accounted for in the calculations by the present morphology model (with (1) the governing equations for the suspended load, the concentration equation (Eq. 16), (2) the boundary condition at $z=b: c=c_{b}(\theta)$ (Eq. 17), and (3) the sediment continuity equation (Eq. 21) with the erosion and deposition terms, $E$ and $D$, given in Eq. 24), presumably resulting in the concentration field illustrated by the sequence of sediment concentration pictures displayed in Figure 12. Although the upwarddirected pressure gradient does not appear explicitly in the aforementioned set of equations, it induces local fluid vertical velocities, and the latter effect contributes to the concentration via the above set of equations controlling the sediment concentration, namely Eqs. 16, 17, 21 and 24. This is clearly observed in Fig. 14 in which local vertical velocity and upward-directed pressure gradient fields in both $y-z$ and $x-z$ planes inside the green vortex given later in Figure 15 (at the vertical located $\mathrm{x}=0.65 D$, and $\mathrm{y}=-0.22 D$ ) are plotted. Here, the vertical distance $z$ is normalized with the sediment size, $d$. As seen from the figure, the upward-directed pressure gradient (positive values of $\left(-\frac{\partial(p / \gamma)}{\partial z}\right)$ ) drives a vertical flow in the upward direction with vigorous velocities of $\mathrm{O}(0.10 \mathrm{~m} / \mathrm{s})$. As a comparison, the fall velocity of a sediment grain of $0.17 \mathrm{~mm}$ in size is $0.02 \mathrm{~m} / \mathrm{s}$, so the sediment cannot settle out of the water column, since its vertical advection by the velocity field in the vortex is much stronger. 

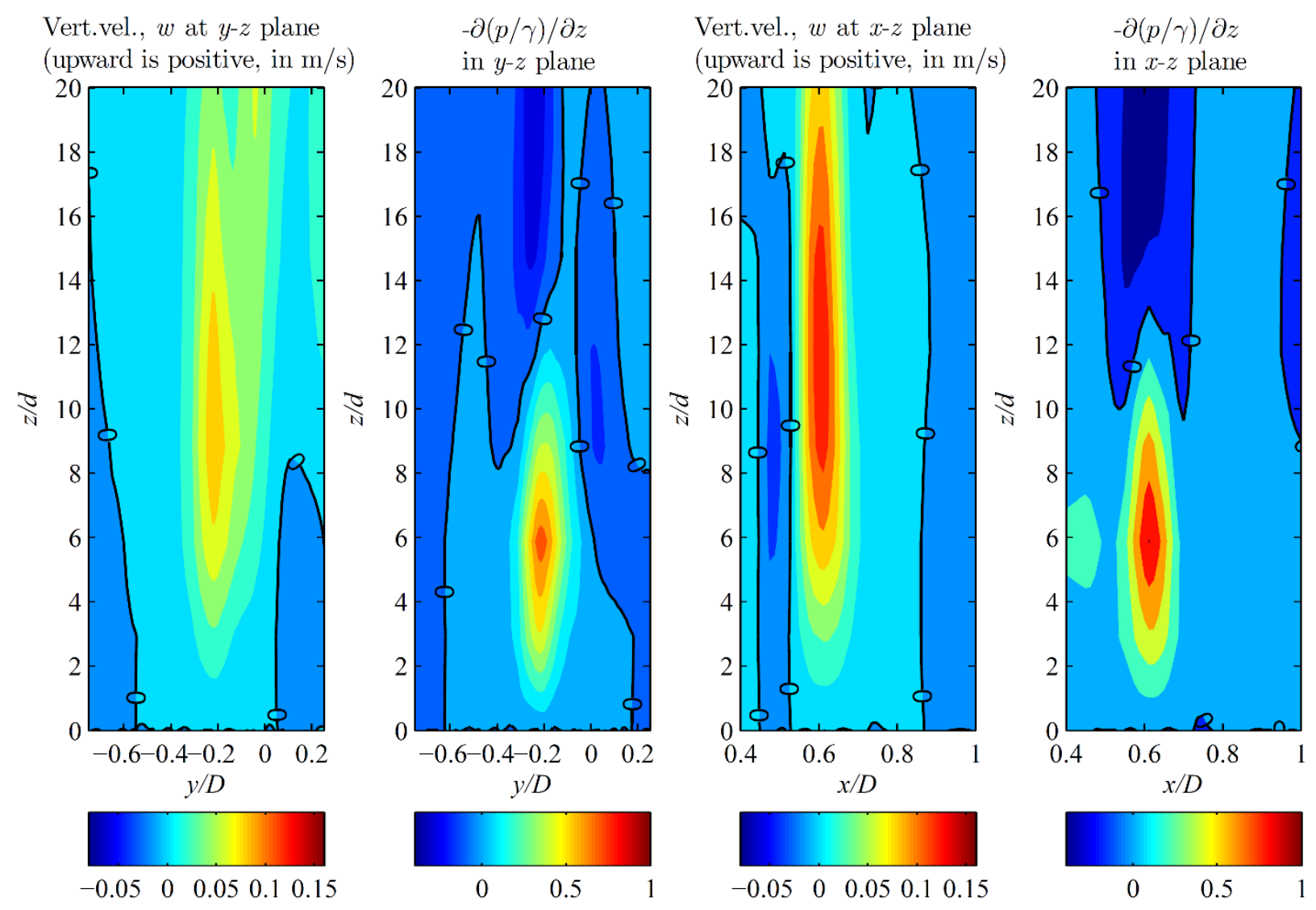

Figure 14. Local vertical velocity $(w)$ and upward-directed pressure gradient $\left(-\frac{\partial(p / \gamma)}{\partial z}\right)$ fields in both $y-z$ and $x-z$ planes inside the green vortex given in Figure 15.

The entrainment of sediment into the flow $(K C=20)$ described above is visualized in an accompanying video (see electronic supplementary material-2 of the paper). The video is composed of three views with the same camera angle looking from downstream to the cylinder. The upper left view depicts the evolution of iso-surfaces of vortical structures created using the Q-criterion (blue transparent structures) and the iso-surfaces of sediment concentration (yellow). The lower left view depicts the Shields parameter computed over the bed using the bed shear stresses only and the iso-surfaces of vortical structures created using the Q-criterion. The right view depicts the evolution of iso-surfaces of vorticity in the $z$-direction (green and blue transparent structures) and the upward-directed pressure gradients $\left(-\frac{\partial(p / \gamma)}{\partial z}\right)=0.1$ (red color structures). The video is given for 20 wave periods. The $Q$-criterion is advantageous in depicting the three dimensional coherent structures such as the horseshoe vortex. However, it does not 
contain directional information for the rotation of the vortices. Use of vorticity in the right view of the video (rather than the $Q$ quantity) is to make the directions of rotation of the lee-wake vortices distinguishable. Here, using a right hand rule, the positive values of vorticity depicted in transparent green color states a counter-clockwise rotation of the vortex, and vice-versa for the blue transparent, negative values.

Figure 15 depicts one final flow visualization regarding the upward directed pressure gradients in which lee-wake vortices in terms of vertical component of the vorticity are depicted (green and orange color transparent structures), and the contour plots of the pressure gradients $\left(-\frac{\partial(p / \gamma)}{\partial z}\right)$ over the sections taken in $x$ and $y$ directions, passing through the two lee-wake vortices, are given. Also, the variation of the pressure gradient in the vertical direction along a vertical line located at one of the locations with the highest observed magnitudes of upward directed pressure gradient, $\left(-\frac{\partial(p / \gamma)}{\partial z}\right) \approx \mathrm{O}(1)$, is given in the figure (the inlet in Figure 15 is to the left). Note that the vertical distance $z$ is normalized here with the sediment size, $d$.

The vertical line (along which the pressure gradient is plotted) is located inside the green colored lee-wake vortex that is just about to be shed. The visualization depicted in Figure 15 corresponds to $\omega t=117^{\circ}(t=67.43 \mathrm{~s})$ of the test described in the previous Figures 12 and 13 . As seen from the figure, the upward-directed pressure gradient, namely $\left(-\frac{\partial(p / \gamma)}{\partial z}\right)>0$, is generated very close to the bed over a vertical distance from just above the bed up to $z / d \approx 10$. 


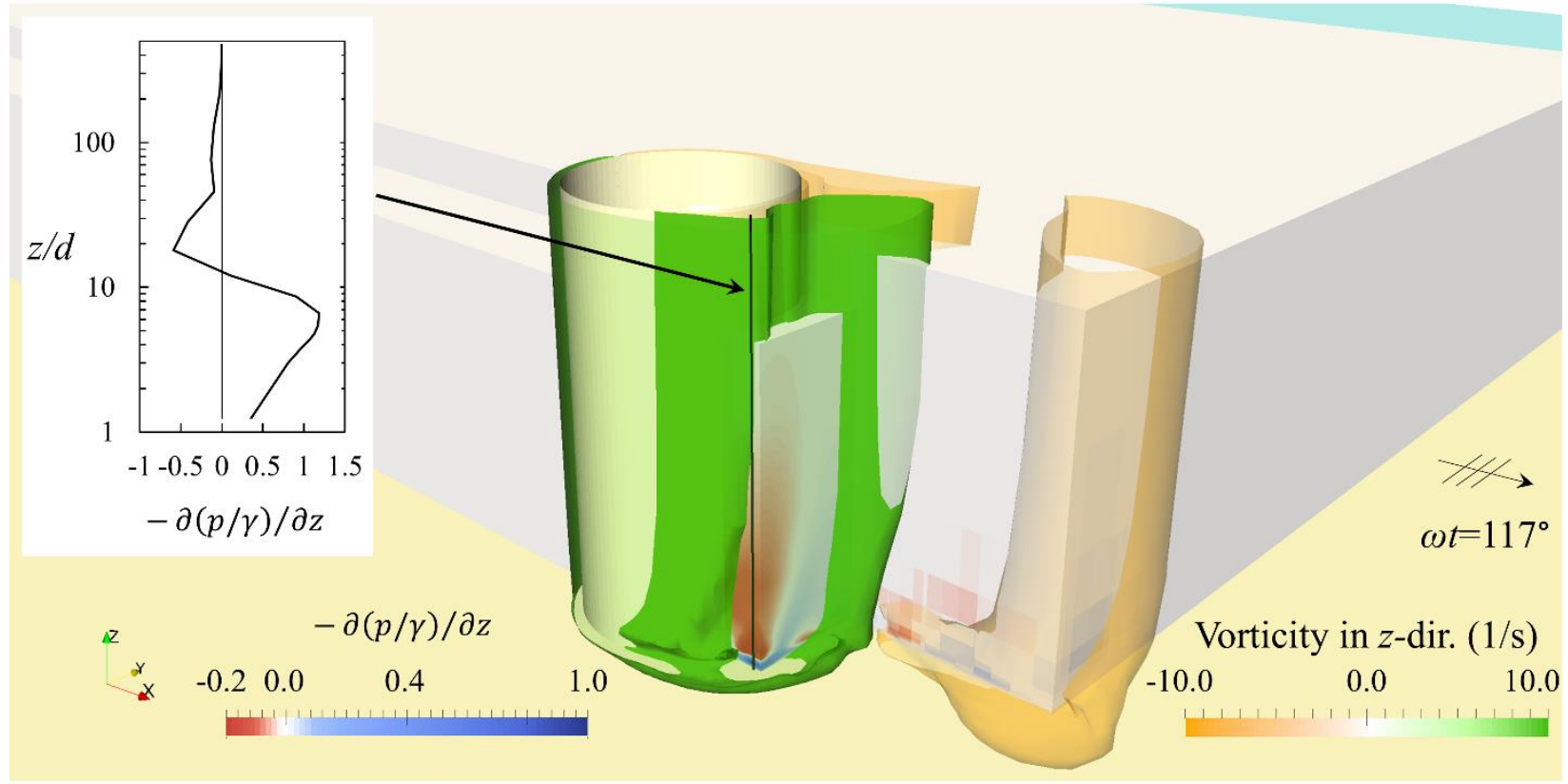

Figure 15. 3D view of upward directed pressure gradients and iso-surfaces of vorticity in $z-$ direction (in $1 / \mathrm{s})$ at $\omega t=117^{\circ}(t=67.43 \mathrm{~s})$ for $K C=20$, Test 14 of Sumer et al.'s (1997) wave flume experiment, given in Table 2.

It should be noted that the aforementioned mechanism of scour in the case of waves is evidently completely different from that responsible for scour in the case of steady currents. In the latter case, the key element of the scour mechanism is the horseshoe vortex (in combination with the contraction of streamlines), as described in Whitehouse (1998), Sumer and Fredsøe (2002), and Roulund et al. (2005) among others. Also, it is important to note that the scour pattern in the case of waves (Figs. 11 and 12) shows no sign of presence of the previously mentioned counterrotating vortices. This is not the case, however, for scour in steady currents. The scour pattern obtained in Roulund et al's (2005, Fig. 33, Panel Time=2:00:00) numerical simulations for steady current clearly exhibits an extension of the scour hole with two elongated scour holes adjacent to the actual scour hole (in the far downstream of the pile) with a longitudinal ridge between them, and the bed form there appeared to be the "signature" of the two counter-rotating vortices, also captured by the simulations of Baykal et al. (2015). The fact that no such scour pattern in the present wave simulations has been observed is clearly an indication of the absence of the two counter-rotating vortices in waves. 
Finally, Figure 16 shows the instantaneous bed shear stress amplification around the pile over the initially plane bed, where the morphology model is not yet switched on, at the phase $\omega t=90^{\circ}$ (Figure 16a); and over the equilibrium scoured bed $\left(\mathrm{t}^{*}=3.5\right.$ ), again, at $\omega t=90^{\circ}$ (Figure 16b) for the $K C=20$ scour case (Test 6 in Table 3). As seen from the figure, the bed shear stress amplification of the equilibrium stage is reduced considerably, apparently from a maximum value of $\left|\tau_{0}\right| / \bar{\tau}_{0 m}=$ 5 (Figure 12.a) to $\left|\tau_{0}\right| / \bar{\tau}_{0 m}=3$ (Figure $12 \mathrm{~b}$ ).
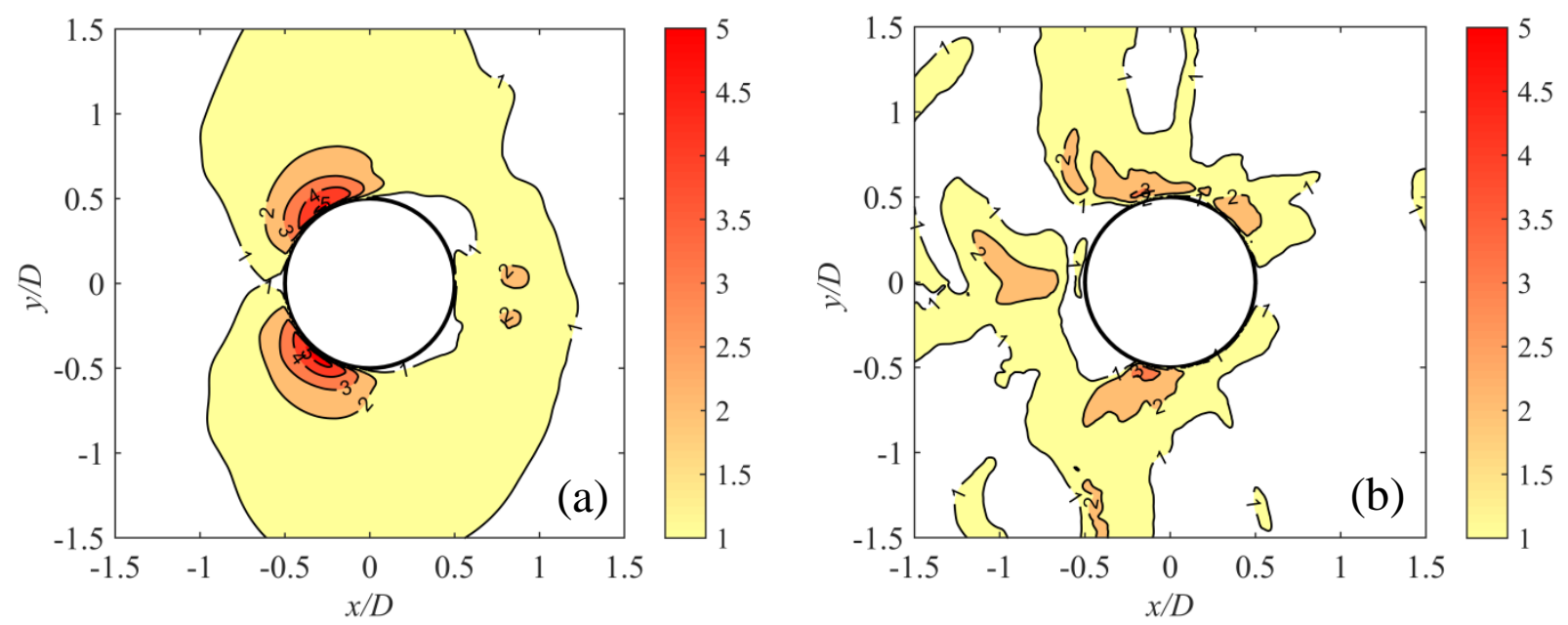

Figure 16. Bed shear stress amplification at $\omega t=90^{\circ}$. Model results for the $K C=20$ scour case, Test 6, Table 3. (a) Initially plain bed. (b) Equilibrium scoured bed at $t^{*}=3.5$.

\section{Backfilling simulations}

The selected backfilling test conditions are based on Sumer et al.'s (2013a) laboratory experiments. These tests are summarized in Table 4, characterized by their initial and final Keulegan-Carpenter numbers, $K C_{i}$ and $K C_{f}$, respectively. Here, $K C_{i}$ corresponds to the flow conditions of the scour test, which is followed by the backfilling test with the flow conditions $K C_{f}$ and the initial bed profile taken as the equilibrium bed profile reached at the end of the scour test. These physical experiments were carried out in irregular waves in Sumer et al. (2013a). The quantities of $U_{m}, T_{w}$, and $U_{f m}$ in Table 4 of the respective $K C_{f}$ are defined in exactly the same way as in Table 3. During the simulation of Test 54 of Sumer et al.'s (2013a) experiments, numerical stability issues were encountered. Therefore, the flow conditions of this test are changed satisfying the same $K C_{f}$ number and Shields parameter $\theta_{f}$ with the original test (the initial $K C_{i}$ and 
$\theta_{i}$ being different); this test is denoted by Test $54^{*}$ (Table 4). We note that the initial scour holes used for the backfilling simulations here are generated by the present scour simulations. To this end, two kinds of scour holes are generated: The scour hole obtained by the scour simulation for Test 1 in Table 2 (the current case, $K C=\infty$ ), and that for Test 6 in Table 3 (the wave case with $K C=20)$.

Table 4. The test conditions for backfilling simulations. All cases use pile diameter $D=40 \mathrm{~mm}$ and sand grain size $d=0.17 \mathrm{~mm}$.

\begin{tabular}{ccccccc}
\hline $\begin{array}{c}\text { Test No. of } \\
\begin{array}{c}\text { Sumer et al.'s } \\
\text { experiments } \\
(2013 \mathrm{a})\end{array}\end{array}$ & $\begin{array}{c}\text { Initial } \\
\text { Keulegan- } \\
\text { Carpenter } \\
\text { number } \\
K C_{i}\end{array}$ & $\begin{array}{c}\text { Final } \\
\text { Keulegan- } \\
\text { Carpenter } \\
\text { number } \\
K C_{f}\end{array}$ & $\begin{array}{c}U_{m} \\
(\mathrm{~m} / \mathrm{s})\end{array}$ & $\begin{array}{c}\text { Wave } \\
\text { period } \\
T_{w}(\mathrm{~s})\end{array}$ & $\begin{array}{c}U_{f m} \\
(\mathrm{~m} / \mathrm{s})\end{array}$ & $\begin{array}{c}\text { Shields } \\
\text { parameter, } \theta_{f}, \\
\text { corresponding to } \\
\text { the final wave } \\
\text { climate }\end{array}$ \\
\hline 27 & $\infty$ (current) & 20 & 0.20 & 4.0 & 0.016 & 0.09 \\
\hline 32 & $\infty$ (current) & 10 & 0.225 & 1.79 & 0.02 & 0.15 \\
\hline 53 & 20 & 11 & 0.175 & 2.5 & 0.017 & 0.10 \\
\hline 52 & 20 & 16 & 0.155 & 4.0 & 0.014 & 0.07 \\
\hline $54 *$ & 20 & 10 & 0.14 & 2.8 & 0.015 & 0.08 \\
\hline
\end{tabular}

${ }^{(1)}: U_{m}$ is the maximum value of the undisturbed orbital velocity at the bed in Sumer et al.'s (2013a) wave-flume experiments (see the text for details), or the maximum value of the free stream velocity of the oscillatory flow in the numerical simulations, defined by Eq. 26, all corresponding to the final wave climate.

(2): $U_{f m}$ is the maximum value of the friction velocity in Sumer et al.'s (2013a) wave-flume experiments, or the maximum value of the friction velocity of the oscillatory flow in the numerical simulations, all corresponding to the final wave climate.

As noticed, the flow is a sinusoidal oscillatory flow in the present scour and backfilling simulations while it is an irregular-wave flow in the corresponding laboratory experiments of Sumer et al. (2013a). Experimenting with a sinusoidal oscillatory flow in numerical simulations has obvious advantages, as opposed to an irregular-wave flow, as it is simple and easy to control, and the end results are easy to verify and validate. Therefore, comparison of the present simulation results with the laboratory experiments of Sumer et al. (2013a) will not be a direct one. Nevertheless, it turns out that the scour depth (in both scour and backfilling processes) appears not to be influenced by the difference in flows in the numerical simulations and in the laboratory experiments of Sumer et al. (2013a). This is not the case, however, for the time scale of the scour and backfilling processes. In order to shed light onto the latter, a supplementary test 
has been carried out in the numerical simulations in which the flow is simulated by the irregular wave flow in Test 32 of Sumer et al.'s (2013) backfilling test, as will be detailed later here in this section.

Backfilling of previously generated scour holes around piles occurs when the flow climate changes into that with milder conditions. Examples include; a scour hole generated by a steady current is backfilled when the flow conditions change from steady current to waves, or to combined waves and current, or from waves to smaller waves, Sumer et al. (2013a). With the present numerical model, several backfilling scenarios have been simulated in which the initial scour hole is generated either by a steady current or by waves (see Table 3), and subsequently it is backfilled with relatively milder waves (see Table 4). The results of two of the backfilling simulations in Table 4, namely Test 27 and Test 32, have been presented in an earlier publication, Baykal et al. (2014). Herein, the final state of these two simulations and three additional backfilling simulations in which the initial scour hole is generated by waves (Table 4) will be presented.

Figure 17 depicts a sequence of pictures, illustrating the time evolution of the backfilling of a scour hole obtained in the present simulations. The scour hole in this simulation is initially generated in Test 1 (Table 3), and the backfilling is achieved by the oscillatory flow in Test 27 (Table 4), with $K C_{f}=20$.

Figure 17 shows that the scour hole, initially generated by the steady current, becomes symmetric in the offshore-onshore direction after 60 wave periods $\left(t^{*}=1.34\right)$. Up to almost this point, the backfilling, for the most part, occurs from the hump at the downstream of the pile. After this first stage (i.e., for times $t^{*}>1.34$ ), a new backfilling regime starts until the new equilibrium scour profile is obtained for this Keulegan-Carpenter number, $K C_{f}=20$.

Figures 18 a-e display the time development of the backfilling process in terms of the depth of the scour hole for the simulated backfilling scenarios (Table 4). The depth of the scour hole plotted in Figure 18 is measured at the offshore edge of the pile. The equilibrium scour depths 
obtained in Sumer et al.'s (2013a) laboratory backfilling experiments are also plotted in Figure 18 (horizontal dashed lines) as reference lines.

The asymptotic values attained by the backfilling time series in Figure 18 (i.e., the equilibrium scour depths, corresponding to the backfilling simulations) are plotted in Figure 19 versus the Keulegan-Carpenter number. In Figure 19, three additional sets of data (corresponding to the equilibrium stage of the processes) are also plotted for comparison, namely: (1) Data from the present scour simulations (see the legend for the symbols); (2) Data from the scour experiments of Sumer et al. (2013a) (empty squares); and (3) Data from the backfilling experiments of Sumer et al. (2013a) (empty circles). The solid line in Figure 19, on the other hand, represents Sumer et al.'s (1992) empirical expression:

$$
\frac{S}{D}=1.3\{1-\exp [-0.03(K C-6)]\} ; K C \geq 6 \text {. }
$$

The laboratory data from Sumer et al. (1992) is not plotted in Figure 19 in order to keep the figure relatively simple. From Figure 18, the data (namely, (1) the scour depths obtained in the present scour simulations, (2) those obtained in the scour experiments of Sumer et al. (1992) represented by the solid curve in Figure 19, (3) those obtained in Sumer et al.'s (2013a) scour experiments, (4) the scour depths from the present backfilling simulations, and (5) those obtained in the backfilling experiments of Sumer et al. (2013a)) all practically collapse on the same curve. This implies that, regardless of the initial scour hole geometry, the equilibrium scour depth of the backfilling process will be the same as that of the ordinary scour process for the same $K C$ number, a result first noted by Sumer et al. (2013a) from comparison of scour and backfilling data from their laboratory experiments. The present numerical simulations confirm this result.

The previously mentioned result has been explained by Sumer et al. (2013a) as follows. Sumer et al.'s (2013a) single particle experiments indicated that the sand particles are eventually brought into the scour hole from outside, in all directions. With the latter result, Sumer et al. (2013a) argued that, being in the scour hole, the sand will subsequently be redistributed by the existing vortex flow around the pile, and that this is precisely the mechanism of the familiar scour process around a pile in waves (Sumer et al., 1992, and 1993). Thus, Sumer et al. (2013a) concluded that the equilibrium stage of the backfilling process should be governed by precisely the same 
mechanism as that causing scour (the mechanism described in conjunction with Figures 12 and 13 earlier). Hence, for a given $K C$ number, the equilibrium scour depth in the backfilling process should be the same as that of the scour process, see Figure 19.

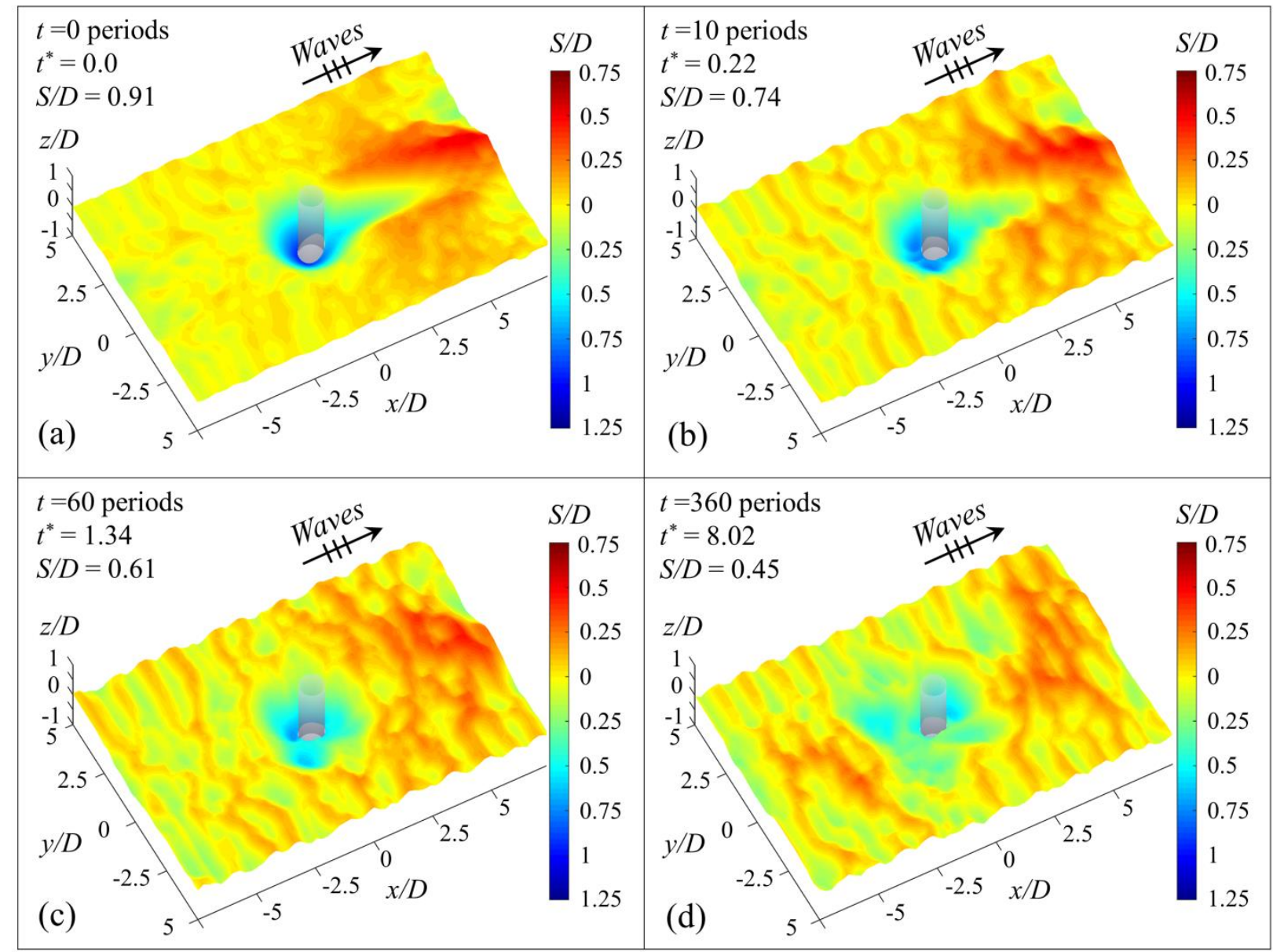

Figure 17. Development of backfilling with waves, $K C=20$ of a scour hole previously generated by a steady current. Numerical simulation. Scour depth indicated in the panels is at the upstream edge of the pile. Test 27, Table 4. 


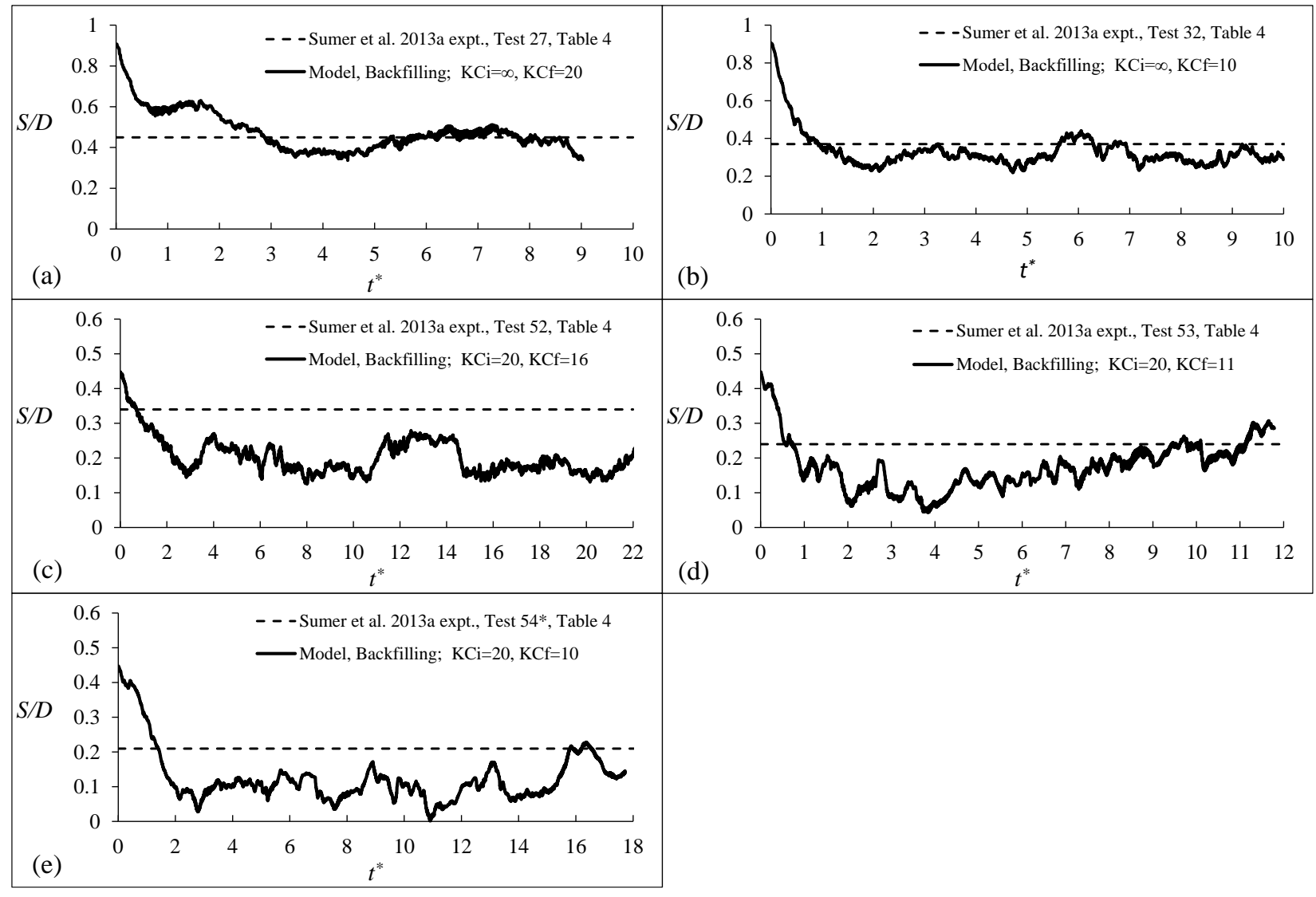

Figure 18. Computed time series of the scour depth at the offshore edge of the pile for the backfilling scenarios (solid line) undertaken in the present simulations. Dashed lines: The equilibrium scour depths obtained for the corresponding tests in Sumer et al.'s (2013a) waveflume experiments. 


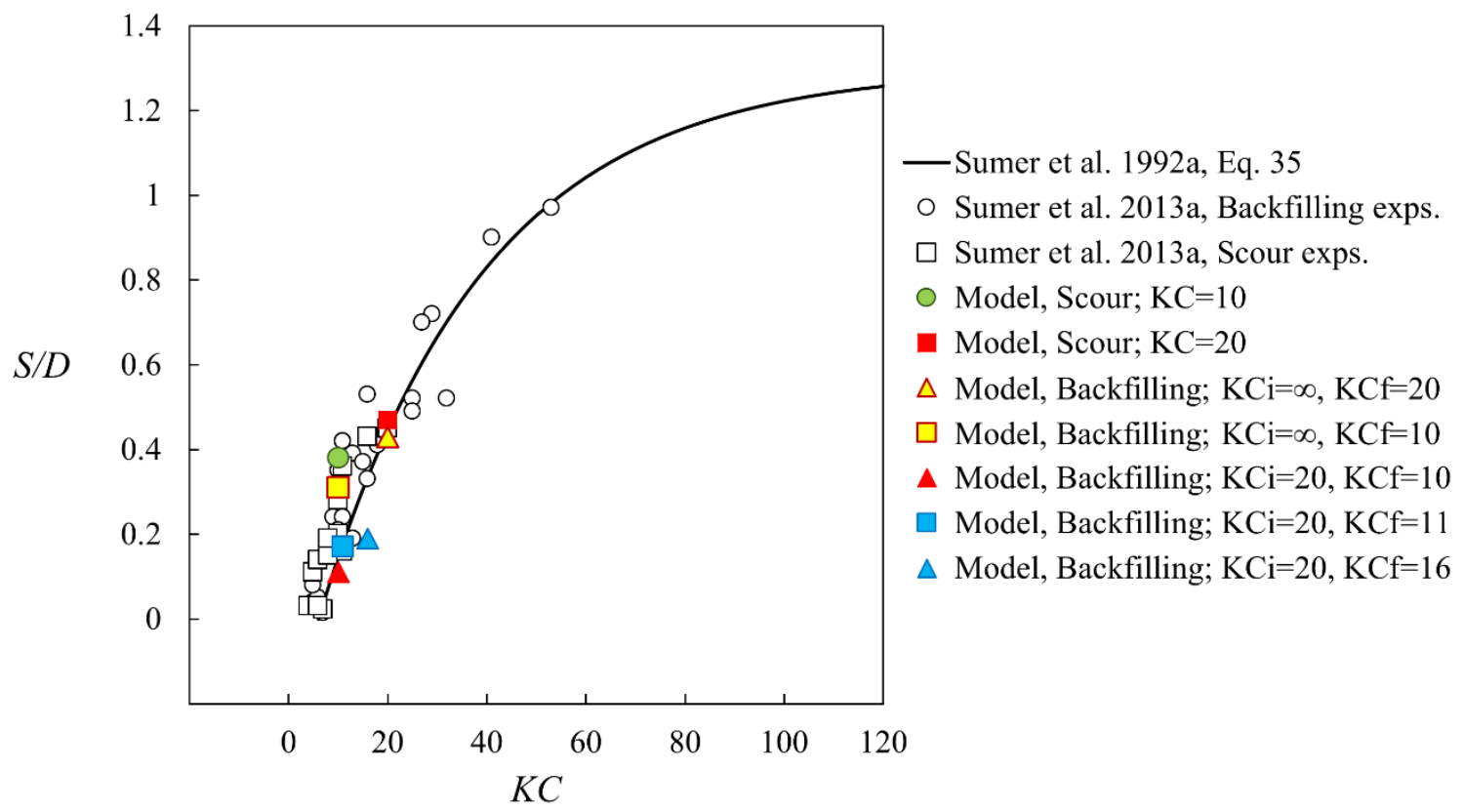

Figure 19. Depth of the scour hole corresponding to the equilibrium stage. Empty squares: From scour experiments of Sumer et al. (2013a). Solid line: Sumer et al.'s (1992) empirical expression (Eq. 35) fitted to the scour data of Sumer et al. (1992). Empty circles: From backfilling experiments of Sumer et al. (2013a). Color symbols: From the present numerical simulations, for both scour and backfilling.

\section{Remarks on prediction of time scale from the numerical simulations}

The time scale of scour (Sumer and Fredsøe, 2002), representing the time required for significant scour to develop, is defined by

$$
S_{t}=S\left[1-\exp \left(-\frac{t}{T}\right)\right],
$$

in which $S_{t}$ is the scour depth at time $t, S$ is the equilibrium scour depth and $T$ is the time scale of the scour process. Similar to the above definition, the time scale for backfilling is defined by

$$
S_{t}=S+\left(S_{i}-S\right) \exp \left(-\frac{t}{T}\right),
$$


in which $S_{t}$ is the depth of the scour hole at time $t, S_{i}$ is the depth of the initial scour hole, and $T$ represents the time period over which a substantial amount of backfilling occurs, the time scale of the backfilling process. Given the time series of the depth of scour hole, $T$ can be readily calculated for both the scour case and the backfilling case.

The time scales calculated in the present simulations are depicted in Table 5. The time scales of Sumer et al.'s (2013a) scour and backfilling experiments corresponding to the present simulations are also provided in Table 5 for comparison. The time scales in the present simulations are calculated from the so-called area method (see, e.g., Sumer and Fredsøe, 2002). Note that, to the authors' experience, a second method, known as the tangent method, gives practically the same results, and therefore not much attention should be given to the method used in the calculations.

Table 5. Summary of the results for the time scale of scour and backfilling cases.

\begin{tabular}{|c|c|c|c|c|c|c|c|}
\hline $\begin{array}{l}\text { Test number } \\
\text { of Sumer et } \\
\text { al.'s (2013a) } \\
\text { current/wave } \\
\text { flume } \\
\text { experiments }\end{array}$ & $\begin{array}{c}\text { Keulegan- } \\
\text { Carpenter } \\
\text { number, } \\
K C\end{array}$ & $\begin{array}{c}\text { Shields } \\
\text { parameter, } \\
\theta\end{array}$ & $\begin{array}{c}\text { Initial } K C \\
\text { number, } \\
\quad K C_{i}\end{array}$ & $\begin{array}{c}\text { Final } \\
K C \\
\text { number, } \\
K C_{f}\end{array}$ & $\begin{array}{c}\text { Final } \\
\text { Shields } \\
\text { parameter, } \\
\theta_{f}\end{array}$ & $\begin{array}{c}\text { Time scale } \\
\text { from Sumer et } \\
\text { al.'s (2013a) } \\
\text { current/wave } \\
\text { flume } \\
\text { experiments, } \\
T^{*}\end{array}$ & $\begin{array}{c}\text { Time scale } \\
\text { from the } \\
\text { present } \\
\text { simulations, } \\
T^{*}\end{array}$ \\
\hline \multicolumn{8}{|c|}{ Scour tests } \\
\hline 1 & $\infty$ (current) & 0.13 & - & - & - & 0.23 & 0.06 \\
\hline 6 & 20 & 0.09 & - & - & - & 2.2 & 1.49 \\
\hline 9 & 10 & 0.15 & - & - & - & 0.5 & 0.46 \\
\hline \multicolumn{8}{|c|}{ Backfilling tests } \\
\hline 27 & - & - & $\infty$ (current) & 20 & 0.09 & 11 & 1.43 \\
\hline 32 & - & - & $\infty$ (current) & 10 & 0.15 & 6.7 & 0.59 \\
\hline 53 & - & - & 20 & 11 & 0.1 & 4.5 & 1.22 \\
\hline 52 & - & - & 20 & 16 & 0.07 & 3.9 & 1.37 \\
\hline $54 *$ & - & - & 20 & 10 & 0.08 & 1.6 & 1.39 \\
\hline
\end{tabular}

Table 5 clearly shows that the time scales from the present simulations are generally significantly smaller than the experimental time scales. For the wave scour (Tests 6 and 9) and the backfilling (Tests 27-54*) experiments, this discrepancy is strongly related to the difference in the waves used in the two studies: The scour and backfilling experiments of Sumer et al. (2013a) were carried out in irregular waves (Eqs. 31-32), whereas the present numerical simulations are achieved in regular waves (Eq. 28). 
It is known that the lee-wake vortex flow regimes are disrupted massively in irregular wave flows, Sumer and Kozakiewicz (1995) (see also Sumer and Fredsøe, 2006, pp. 322-325). Sumer and Kozakiewicz's study (1995, Fig. 2) shows that the regular vortex shedding regime is disrupted tremendously when the flow changes from the regular oscillatory flow to an irregular one, with a value of spectral-width parameter of 0.56 , the value typical for JONSWAP wave spectrum, used in Sumer et al.'s (2013a) experiment. In more quantitative terms, Fig. 2 of Sumer and Kozakiewicz's study (1995) does indicate that the regular vortex shedding regime in the case of the irregular oscillatory flow occurs only over about $25 \%$ of the time while the so-called vortex-pair regime (with $\mathrm{O}(1)<K C_{\text {temporal }}<7$ ) occurs practically over the rest of the time.

Now, from wave scour studies, the vortex-pair regime is associated with no net scour (see, e.g., Sumer and Fredsøe, 2002), and therefore the consequence of experimenting with irregular waves is that the time scale will be significantly increased. This is essentially what happens with regard to the time-scale results of Sumer et al.'s (2013a) wave experiments in Table 5 (Tests 6-54*).

In response to one of the reviewers' comments regarding the time scale, a supplementary test has been carried out for Test 32 (Table 4) where the flow was simulated as an irregular-wave flow with randomly generated velocities at the inlet following a JONSWAP shaped spectrum with spectral width parameter 0.56, identical to the irregular waves in Sumer et al.'s (2013a) experiment. The calculations have so far advanced for times $0<t *<4$. The time development of backfilling obtained in this supplementary numerical test is presented in Figure 20 along with that calculated for the same test with regular oscillatory flow, reproduced from Figure 18b. Figure 20 clearly shows that the time scale is increased tremendously when the flow is changed from regular oscillatory flow to irregular one. The figure indicates a time scale of the order of magnitude of $T^{*}=\mathrm{O}(3-4)$, which is not radically different from the experiment, $\mathrm{O}(6-7)$ (Table 5). 


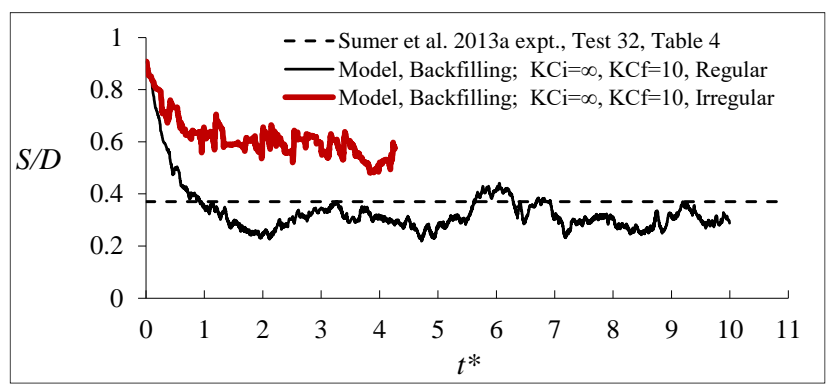

Figure 20. Computed time series of the scour depth at the offshore edge of the pile for the backfilling scenario Test 32 given in Table 4 with regular oscillatory flow (thin black solid line) and irregular oscillatory flow (thick red line) undertaken in the present simulations. Dashed line: The equilibrium scour depth obtained for the corresponding test in Sumer et al.'s (2013a) waveflume experiments.

In addition to this important effect, the following can also partly account for the observed discrepancy for the tests where the initial scour hole is generated by current (Tests 27 and 32). The current scour test carried out in Tests 27 and 32 in Sumer et al. (2013a) was conducted for very large times, so large that the accumulated sand at the downstream of the pile was washed away. Therefore, the backfilling in Sumer et al.'s (2013a) experiments in Tests 27 and 32 took place much more gradually, causing an additional increase in the time scale of backfilling for these tests.

The preceding paragraphs have discussed the wave cases. As for the current case (Test 1, Table 5), the discrepancy in the time scale between Sumer et al.'s (2013a) laboratory experiment and the present simulation may, partly, be explained in terms of the water-depth-to-pile-diameter ratio, $\delta / D$, one of the key parameters influencing the scour depth in currents. The scour depth increases with increasing $\delta / D$ (Sumer and Fredsøe, 2002) due to the increase in the horizontal extent of the horseshoe vortex with increasing $\delta / D$. The scour depth eventually attains a constant value as $\delta / D \rightarrow \infty$. Now, the boundary-layer-thickness-to-diameter ratio was $\delta / D=2$ in the numerical simulation, while it was $\delta / D=10$ in Sumer et al.'s (2013a) experiment. From the scour-depth-versus- $\delta / D$ data (Sumer and Fredsøe, 2002), the scour depth is about $15-20 \%$ smaller in the case of $\delta / D=2$ (the present numerical simulation) than in the case of $\delta / D=10$ (Sumer et al.'s experiment). The latter implies that the time scale in the numerical simulation should be 
somewhat smaller than in the case of Sumer et al. (2013a), consistent with the comparison displayed in Table 5 for Test 1.

One final remark regarding the observed discrepancy in Table 5 is the following. In the present numerical tests, the flow in the backfilling tests is impulsively started (save the part corresponding to a "ramp-up" time, typically some wave periods). In Sumer et al.’s (2013a) experiments, the flow was not started impulsively, but rather it developed during the course of a ramp-up time, which was $\mathrm{O}\left(10 T_{w}\right)$. This also would result in time scales larger than that without a ramp-up time (the present simulation). Nevertheless, as described in the preceding paragraphs, the major reason for the observed discrepancy for the wave cases is the difference between the waves in the tests, namely that the laboratory experiments were conducted in irregular waves whereas the numerical simulations were conducted in regular waves.

It may be noted that the equilibrium scour depth in waves is controlled by the length of the leewake flow, and it is not affected by whether or not the waves are regular or irregular, as revealed by Figure 19. This is simply because the length of the exposure area in the lee wake for large times in the case of irregular waves will be practically the same as in regular waves for the same Keulegan-Carpenter number.

\section{Conclusions}

The conclusions drawn from the present numerical simulations are summarized as follows.

1. The horseshoe vortex, the lee-wake flow and the pair of two counter-rotating vortices, Figure 1, (the principal features of flow around a circular pile) undergo substantial changes when the flow is changed from steady current to waves. In the case of waves, the horseshoe vortex exists only for a certain portion of the half period of the wave flow, and with a somewhat diminished strength. This result is in complete agreement with laboratory observations. Likewise, the leewake flow also undergoes significant changes. The two-cell structure of vortex shedding observed for steady currents practically disappears in the case of waves. In the latter case, the vortex-shedding regimes appear to be very similar to those for free cylinders. This is essentially 
due to the small wave-boundary-layer thickness in the case of waves. The pair of two counterrotating vortices further downstream of the lee-wake, observed for steady currents, is practically non-existent in the case of waves, also due to the small wave-boundary-layer thickness in this latter case.

2. The way in which scour occurs in the case of waves has been numerically visualized, according to which the shed vortex picks up the sediment and sweeps it into its core, and the sediment is carried away from the pile, as the vortex is convected downstream; the sediment is eventually deposited on the bed when the vortex ceases to exist, resulting in a net scour around the pile. This result is in complete agreement with experimental observations. This mechanism is in stark contrast to that for scour in steady currents where the major flow features controlling the scour is the horseshoe vortex in combination with the contraction of streamlines. The numerical results indicate that, in waves, the immense concentration of the bed shear stress and the upwarddirected pressure gradient created underneath the shed vortices are the key elements of the scour mechanism.

3. The present numerical model has proven successful in predicting backfilling of scour holes exposed to waves. The results of the backfilling tests indicate that the equilibrium depth of scour holes is the same for both the scour process and the backfilling process for a given KeuleganCarpenter number, in full agreement with the existing experimental information. (The KeuleganCarpenter number in the case of irregular waves is based on the maximum value of the undisturbed orbital velocity at the bed, defined by Eqs. 31 and 32, and the peak period.) The backfilling of a scour hole is eventually governed by the same mechanism as that of scour referred to in item 2 above.

\section{Acknowledgements}

The first three authors acknowledge the support from FP7-ENV-2013.6.4-3 European Union project ASTARTE (Assessment, STrategy And Risk Reduction for Tsunamis in Europe), Grant No. 603839. The first author also acknowledges the support of a post-doctoral grant from The Scientific and Technical Research Council of Turkey (TUBITAK, Grant No. 2219). The first and 
second author additionally acknowledge Innovative Multi-purpose Offshore Platforms: Planning, Design and Operation (MERMAID), 2012-2016, Grant Agreement No. 288710 of European Commission, 7th Framework Programme for Research.

\section{References}

An, H., Cheng, L. and Zhao, M., 2015. Two-dimensional and three-dimensional simulations of oscillatory flow around a circular cylinder. Ocean Engineering, 109, 270-286.

Baranya, S., Olsen, N.R.B., Stoesser, T. and Sturm, T.W., 2012. Three-dimensional RANS modelling of flow around circular piers using nested grids. Eng. Appl. Comput. Fluid Mech. 6, 648-662.

Baykal, C., Fuhrman, D.R., Sumer, B.M., Jacobsen, N.G. and Fredsøe, J., 2015. Numerical investigation of flow and scour around a vertical circular cylinder. Phil. Trans. R. Soc. A, article no. 20140104. Theme issue 'Advances in fluid mechanics for offshore engineering: a modelling perspective'. compiled and edited by Maurizio Brocchini and Odd Magnus Faltinsen, 28 January 2015; volume 373, issue 2033. (doi: 10.1098/rsta.2014.0104)

Baykal, C., Fuhrman, D.R., Sumer, B.M., Jacobsen, N.G. and Fredsøe. J., 2014. Numerical modeling of backfilling process around monopiles. In Proc. 34th Int. Conf. on Coastal Engineering, Seoul, Korea, 15-20 June 2014. (doi: 10.9753/icce.v34.sediment.22)

Brøker, I.H., 1985. Wave Generated Ripples and Resulting Sediment Transport in Waves, Series Paper No. 36, ISVA. Technical University of Denmark.

Byrne, B.W. and Houlsby G.T., 2003. Foundations for offshore wind turbines. Phil. Trans. R. Soc. A 361, 2909-2930. (doi:10.1098/rsta.2003.1286)

Cebeci, T. and Chang, K.C., 1978. Calculation of incompressible rough-wall boundary-layer flows. AIAA J. 16, 730-735. (doi:10.2514/3.7571)

Dixen, M., Sumer, B.M. and Fredsøe J., 2013. Numerical and experimental investigation of flow and scour around a half-buried sphere. Coast. Eng. 73, 84-105. (doi:10.1016/j.coastaleng.2012.10.006)

Engelund, F. and Fredsøe, J., 1976. A sediment transport model for straight alluvial channels. Nordic Hydrol. 7, 293-306. 
Engelund, F. and Fredsøe, J., 1982. Hydraulic theory of alluvial rivers. Adv. Hydrosci. 13, 187215. (doi:10.1016/B978-0-12-021813-4.50009-3)

Engelund, F., 1970. A note on the mechanics of sediment suspension. Basic Res. Prog. Rep. No. 21, Hydraulic Lab. Tech. Univ. Denmark, 7-14.

Escauriaza, C. and Sotiropoulos, F., 2011a. Initial stages of erosion and bed form development in a turbulent flow around a cylindrical pier. J. Geophys. Res. 116, F03007.

Escauriaza, C. and Sotiropoulos, F., 2011b. Lagrangian model of bed-load transport in turbulent junction flows. J. Fluid Mech. 666, 36-76.

Escauriaza, C. and Sotiropoulos, F., 2011c. Reynolds number effects on the coherent dynamics of the turbulent horseshoe vortex system, Flow Turbul. Combust. 86,2, 231-262.

Fredsøe, J. and Deigaard, R., 1992. Mechanics of coastal sediment transport. World Scientific Publishing, Singapore.

Fuhrman, D.R., Schløer, S. and Sterner, J., 2013. RANS-based simulation of turbulent wave boundary layer and sheet-flow sediment transport processes. Coast. Eng. 73, 151-166.

Fuhrman, D.R., Baykal, C., Sumer, B.M., Jacobsen, N.G. and Fredsøe, J., 2014. Numerical simulation of wave-induced scour and backfilling processes beneath submarine pipelines. Coastal Eng. 94, 10-22.

Gothel, O. and Zilke, V., 2006. Numerical modeling of scour at offshore wind turbines. In Proc. 30th Int. Conf. on Coastal Engineering, San Diego, CA, 3-8 September 2006, 3, 2343-2353, Singapore: World Scientific.

Gothel, O., 2008. Numerical modeling of flow and wave-induced scour around vertical structures. PhD thesis, Leibniz University of Hanover, Faculty of Civil Engineering and Geodesy, Hanover, Germany.

Harris, J.M., Whitehouse, R.J.S. and Benson, T., 2010. The time evolution of scour around offshore structures. Maritime Eng., 163 (MA1), 3-17.

Hjelmfelt, A. T. and Lenau, C. W., 1970. Nonequilibrium Transport of Suspended Sediment. Journal of the Hydraulics Division - ASCE, 96, 1567-1586.

Hjorth, P., 1975. Studies on the nature of local scour. Lund Institute of Technology, University of Lund, Bulletin Series A, no. 46, viii edn. Lund, Sweden: Department of Water Resources Engineering. 1. 
Hunt, J. C. R., Wray, A. and Moin, P., 1988. Eddies, stream, and convergence zones in turbulent flows. Center for Turbulence Research Report, CTR-S88.

Issa, R.I., 1986. Solution of implicitly discretized fluid flow equations by operator-splitting. J. Comput. Phys., 62, 40-65.

Jacobsen, N.G., 2011. A full hydro- and morphodynamic description of breaker bar development. PhD thesis, Technical University of Denmark, Kongens Lyngby, Denmark.

Jacobsen, N.G., Fredsøe, J. and Jensen, J.H., 2014a. Formation and development of a breaker bar under regular waves. Part 1. Model description and hydrodynamics. Coast. Eng. 88, 182193. (doi:10.1016/j.coastaleng.2013.12.008)

Jacobsen, N.G., van Velzen, G. and Fredsøe, J., 2014b. Analysis of pile scour and associated hydrodynamic forces using proper orthogonal decomposition. In Proc. 7th Int. Conf. on Scour and Erosion, Perth, Australia, 2.4 December 2014. Leiden, The Netherlands: CRC Press/Balkema.

Jacobsen, N.G., 2015. Mass conservation in computational morphodynamics: uniform sediment and infinite availability. International Journal for Numerical Methods in Fluids, 78, 233256.

Jasak, H. and Tuković, Ž., 2006. Automatic mesh motion for the unstructured finite volume method. Transactions of FAMENA 30 (2), 1-20.

Jensen, B.L., Sumer, B.M. and Fredsøe, J. 1989. Turbulent oscillatory boundary layers at high Reynolds numbers. J. Fluid Mech., 206, 265-297.

Jensen, K.L., Sumer, B.M., Vittori, G. and Blondeaux, P., 2014. Role of vertical pressure gradient in wave boundary layers. In Proc. 34th Int. Conf. on Coastal Engineering, Seoul, Korea, 15-20 June 2014. (doi: 10.9753/icce.v34.waves.47)

Justesen, P., Fredsøe, J. and Deigaard, R., 1986. The bottleneck problem for turbulence in relation to suspended sediment in the surf zone. In Proc. 20th Int. Conf. on Coastal Engineering, Conference, Taipei, 2, 1225-1239.

Kirkil, G. and Constantinescu S.G., 2005. The horseshoe vortex system around a circular bridge pier on a flat bed. In Proc. 31st International Association Hydraulic Research Congress: Water Engineering for the Future, Choices and Challenges, Seoul, Korea, 11-16 September 2005. Seoul, Korea: KoreaWater Resources Association. 
Kirkil, G., Constantinescu, S.G. and Ettema, R., 2005. The horseshoe vortex system around a circular bridge pier on equilibrium scoured bed. In Proc. World Water and Environmental Resources Congress: Impacts of Global Climate Change, Anchorage, Alaska, 15-19 May 2005, 1-12. Red Hook, NY: ASCE/Curran Associates, Inc.

Kirkil, G., Constantinescu, G. and Ettema, R., 2006. Investigation of the velocity and pressure fluctuations distributions inside the turbulent horseshoe vortex system around a circular bridge pier. In River Flow 2006, pp. 709-718. Taylor \& Francis/A. A. Balkema.

Kirkil, G., Constantinescu, G. and Ettema, R., 2008. Coherent structures in the flow field around a circular cylinder with scour hole. J. Hydrol. Eng. 134, 572-587.

Liang, D. and Cheng, L., 2005. Numerical model for wave-induced scour below a submarine pipeline. J. Waterway, Port, Coastal, Ocean Eng. ASCE, Vol.131, pp193-202.

Liu X. and Garcia M.H., 2008. Three-dimensional numerical model with free water surface and mesh deformation for local sediment scour. J. Waterw. Port C-ASCE 134, 203.217. (doi:10.1061/(ASCE)0733-950X(2008)134:4(203))

Nielsen, A.W., and Hansen, E.A., 2007. Time varying wave and current induced scour around offshore wind turbines. In Proc., 26th ASME 2007 26th Int. Conf. on Offshore Mechanics and Arctic Engineering, Offshore Mechanics and Arctic Engineering (OMAE), San Diego, 399-408.

Olsen, N.R.B. and Kjellesvig, H.M., 1998. Three-dimensional numerical flow modeling for estimation of maximum local scour depth. J. Hydraul. Eng. ASCE 36, 579-590. (doi:10.1080/00221689809498610)

Olsen, N.R.B. and Melaaen, M., 1993. Three-dimensional calculation of scour around cylinders. J. Hydraul. Eng. ASCE, 119, 1048-1054. (doi:10.1061/(ASCE)0733-9429(1993)119:9(1048))

OpenFOAM $^{\circledR}$, 2010. The OpenSource CFD Toolbox, Version 1.6-ext by The OpenFOAM ${ }^{\circledR}$ Extend Project Group, November 12th, 2010 (related websites: http://www.openfoam.org and http:// www.extend-project.de).

Paik, J., Escauriaza, C. and Sotiropoulos, F., 2007. On the bimodal dynamics of the turbulent horseshoe vortex system in a wing-body junction. Phys. Fluids 19 (4), 115104. 
Patankar, S.V. and Spalding, D.B., 1972. A calculation procedure for the heat, mass and momentum transfer in three-dimensional parabolic flows, Int. J. Heat and Mass Transfer $15,1972,1787-1805$

Petersen, T.U., 2014. Scour around offshore wind turbine foundations. PhD thesis, Technical University of Denmark, Kongens Lyngby, Denmark.

Petersen, T.U., Sumer, B.M., Fredsøe, J., Raaijmakers, T.C. and Schouten, J.-J., 2015. Edge scour at scour protections around piles in the marine environment - Laboratory and field investigation, Coast. Eng., 106, 42-72. (doi: 10.1016/j.coastaleng.2015.08.007)

Raaijmakers, T. and Rudolph, D., 2008a. Time-dependent scour development under combined current and wave conditions-Laboratory experiments with online monitoring technique. In Proc. 4th Int. Conf. Scour Erosion, ICSE, Tokyo, 152-161.

Raaijmakers, T. and Rudolph, D., 2008b. Time-dependent scour development under combined current and wave conditions-Hindcast of field measurements. In Proc. 4th Int. Conf. Scour Erosion, ICSE, Tokyo, 340-347.

Roulund, A., Sumer, B.M., Fredsøe, J. and Michelsen, J., 2005. Numerical and experimental investigation of flow and scour around a circular pile. J. FluidMech. 534, 351-401. (doi: $10.1017 / \mathrm{S} 0022112005004507)$

Schlichting, H., 1979. Boundary layer theory, 7th Ed., McGraw Hill, New York.

Stahlmann, A. and Schlurmann, T., 2012. Investigations on scour development at tripod foundations for offshore wind turbines: modeling and application, In Proc. 33rd Int. Conf. on Coastal Engineering. (doi:10.9753/icce.v33.sediment.90)

Stahlmann, A., 2014. Numerical and experimental modeling of scour at foundation structures for offshore wind turbines. J. Ocean Wind Energy, 1, 82-89.

Sumer, B.M., 1984. Lift forces on moving particles near boundaries, Journal of Hydraulic Engineering, ASCE, 110 (9), 1272-1278.

Sumer, B.M., 2014. Liquefaction Around Marine Structures. World Scientific, 472 pp.

Sumer, B.M. and Bakioglu, M., 1984. On the formation of ripples on an erodible bed. Journal of Fluid Mechanics, Vol. 144, 177-190.

Sumer, B.M. and Kozakiewicz, A., 1995. Visualization of flow around cylinder in irregular waves. Int. J. of Offshore and Polar Eng., 5 (4), 270-272. 
Sumer, B.M. and Fredsøe, J., 2002. The Mechanics of Scour in the Marine Environment. World Scientific Publishing.

Sumer, B.M. and Fredsøe, J., 2006. Hydrodynamics around cylindrical structures. World Scientific Publishing. First edition 1997.

Sumer, B.M., Fredsøe, J. and Christiansen, N., 1992. Scour around a vertical pile in waves. J. Waterway, Port, Coastal, Ocean Eng., ASCE, 118(1), 15-31.

Sumer, B.M., Christiansen, N. and Fredsøe, J., 1993. Influence of cross section on wave scour around piles. J. Waterway, Port, Coastal and Ocean Eng., ASCE, 119 (5), 477-495.

Sumer, B.M., Christiansen, N. and Fredsøe, J., 1997. The horsehose vortex and vortex shedding around a vertical wall-mounted cylinder exposed to waves. J. Fluid Mech., 332, 41-70.

Sumer, B.M., Hatipoglu, F., Fredsøe, J. and Sumer, S.K., 2006. The sequence of soil behaviour during wave-induced liquefaction. Sedimentology, 53, 611-629.

Sumer, B.M., Sen, M.B., Karagali I., Ceren B., Fredsøe J., Sottile, M., Zilioli, L., and Fuhrman, D.R., 2011. Flow and Sediment transport induced by a plunging solitary wave. J. Geophys. Res., 116, C01008, doi:10.1029/2010JC006435, 1-15.

Sumer, B.M., Petersen, T.U., Locatelli, L., Fredsøe, J., Musumeci, R.E. and Foti, E., $2013 a$. Backfilling of a scour hole around a pile in waves and current. J. Waterway, Port, Coastal, Ocean Eng., ASCE, 139, 9-23.

Sumer, B.M., Guner, H.A.A., Hansen, N.M., Fuhrman, D.R. and Fredsøe, J., 2013b. Laboratory observations of flow and sediment transport induced by plunging regular wave. J. Geophysical Research: Oceans, 118, 1-22, (doi:10.1002/2013JC009324).

Sumer, B.M., Baykal, C., Fuhrman, D.R., Jacobsen, N.G., Fredsøe, J., 2014. Numerical calculation of backfilling of scour holes. Scour and Erosion (ISBN: 978-1-138-02732-9), pages: 633-642, 2014, C R C Press LLC, Presented at: 7th international Conference on Scour and Erosion, 2014, Scarborough, Western Australia.

van Driest, E.R., 1956. On turbulent flow near a wall. J. Aeronautical Sciences 23 (11), 10071011. (doi:10.2514/8.3713)

van Rijn, L.C., 1984. Sediment transport. Part II. Suspended load transport, J. Hydraulic Engineering, ASCE, 110 (11), 1613-1641.

Vanoni, V.A. (ed.), 1975. Sedimentation Engineering. Manuals and reports on engineering practice; no. 54, ISBN 0-7844-0823-8. 
Whitehouse, R. J. S., 1998. Scour at marine structures, Thomas Telford, London.

Wilcox, D.C., 2006. Turbulence modeling in CFD, 3rd Edition. DCW Industries, Inc., La Canada, California.

Wilcox, D.C., 2008. Formulation of the k- $\omega$ turbulence model revisited. AIAA J. 46, 2823-2838.

Williamson, C.H.K., 1985. Sinusoidal flow relative to circular cylinders. J. Fluid Mech., 155, 141-174.

Zhao, M. and Cheng, L., 2008. Numerical modeling of local scour below a piggyback pipeline in currents. J. Hydraul. Eng. ASCE, 134, 1452-1463. (doi:10.1061/(ASCE)0733-9429 (2008)134:10(1452)).

Zhao, M., Cheng, L., and Zang, Z., 2010. Experimental and numerical investigation of local scour around a submerged vertical circular cylinder in steady currents, Coast. Eng., 57, 709-721. (doi:10.1016/j.coastaleng.2010.03.002). 